TITLE:

\title{
Functional characterization of olfactory receptors in the Oriental fruit fly Bactrocera dorsalis that respond to plant volatiles
}

\section{$\operatorname{AUTHOR}(S)$ :}

Miyazaki, Hitomi; Otake, Jun; Mitsuno, Hidefumi; Ozaki, Katsuhisa; Kanzaki, Ryohei; Chui-Ting Chieng, Anna; KahWei Hee, Alvin; Nishida, Ritsuo; Ono, Hajime

\section{CITATION:}

Miyazaki, Hitomi ...[et al]. Functional characterization of olfactory receptors in the Oriental fruit fly Bactrocera dorsalis that respond to plant volatiles. Insect biochemistry and molecular biology 2018, 101: 32-46

\section{ISSUE DATE:}

2018-10

URL:

http://hdl.handle.net/2433/235533

\section{RIGHT:}

(C) 2018. This manuscript version is made available under the CC-BY-NC-ND 4.0 license

http://creativecommons.org/licenses/by-nc-nd/4.0/:; The full-text file will be made open to the public on 1 October 2019 in accordance with publisher's 'Terms and Conditions for Self-Archiving'.; この論文は出版社版でありません。引 用の際には出版社版をご確認ご利用ください。; This is not the published version. Please cite only the published version. 
1 Functional characterization of olfactory receptors in the Oriental

2 fruit fly Bactrocera dorsalis that respond to plant volatiles

3 Hitomi Miyazaki ${ }^{\mathrm{a}, 1}$, Jun Otake ${ }^{\mathrm{a}}$, Hidefumi Mitsuno ${ }^{\mathrm{b}}$, Katsuhisa Ozaki ${ }^{\mathrm{c}}$, 4 Ryohei Kanzaki ${ }^{\text {b }}$, Anna Chui-Ting Chieng, , Alvin Kah-Wei Heed, Ritsuo

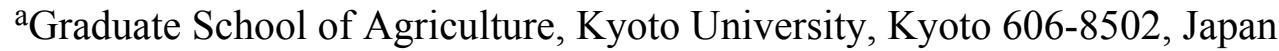

${ }^{b}$ Research Center for Advanced Science and Technology, University of Tokyo, Tokyo 153-

9 8904, Japan

10 'JT Biohistory Research Hall, Takatsuki Osaka, 569-1125, Japan

11 dFaculty of Science, Universiti Putra Malaysia, 43400 UPM Serdang, Malaysia

12

13

14

15

16

$17{ }^{*}$ Corresponding author. Division of Applied Life Sciences, Graduate School of

18

19

20

21

22

23 


\section{ABSTRACT}

25 The Oriental fruit fly, Bactrocera dorsalis, is a highly destructive pest of various

26 fruits. The reproductive and host-finding behaviors of this species are affected by

27 several plant semiochemicals that are perceived through chemosensory receptors.

28 However, the chemosensory mechanisms by which this perception occurs have not

29 been fully elucidated. We conducted RNA sequencing analysis of the chemosensory

30 organs of $B$. dorsalis to identify the genes coding for chemosensory receptors. We

31 identified 60 olfactory receptors (ORs), 17 gustatory receptors and 23 ionotropic

32 receptors - including their homologs and variants - from the transcriptome of male

33 antennae and proboscises. We functionally analyzed ten ORs co-expressed with the

34 obligatory co-receptor ORCO in Xenopus oocytes to identify their ligands. We tested

3524 compounds including attractants for several Bactrocera species and volatiles from

36 the host fruits of $B$. dorsalis. We found that BdorOR13a co-expressed with ORCO

37 responded robustly to 1-octen-3-ol. BdorOR82a co-expressed with ORCO responded

38 significantly to geranyl acetate, but responded weakly to farnesenes (a mixture of

39 isomers) and linalyl acetate. These four compounds were subsequently subjected to

40 behavioral bioassays. When each of the aforementioned compound was presented in

41 combination with a sphere model as a visual cue to adult flies, 1-octen-3-ol, geranyl

42 acetate, and farnesenes significantly enhanced landing behavior in mated females, but

43 not in unmated females or males. These results suggest that the ORs characterized in

44 the present study are involved in the perception of plant volatiles that affect host-

45 finding behavior in $B$. dorsalis.

46

47 Keywords: Chemosensory receptor; Bactrocera dorsalis; Plant semiochemical;

$48 \quad$ Functional analysis; Xenopus oocyte; Behavioral bioassay 
51 Plant semiochemicals play a crucial role in insect-plant interactions, because they

52 affect insect physiology and behavior (Reddy and Guerrero, 2004). Many

53 phytophagous insects use plant semiochemicals as cues to find their feeding, mating,

54 and oviposition sites. Moreover, some insects specifically recognize host plant

\section{Introduction}

$$
\text { chemicals via chemosensory organs to acquire or sequester those chemicals as }
$$

defensive substances, sex pheromones, or sex pheromone precursors (Nishida, 2002;

Opitz and Müller, 2009). Therefore, an elucidation of the basic mechanisms

underlying the chemoreception of plant semiochemicals is essential for an

understanding of the adaptation of phytophagous insects to plants as sources of essential substances for growth and reproduction.

The tephritid fruit fly species, which include destructive horticultural pests in both tropical and temperate regions, provide a good model for understanding how insects adapt to plant chemicals, because their life cycles involve response to several characteristic semiochemicals (Metcalf, 1990; Shelly, 2010). Several such species belonging to two Dacinae genera, Bactrocera and Zeugodacus, exhibit striking behaviors towards certain semiochemicals that contribute to the floral fragrances of several orchid species. For example, male Oriental fruit flies (Bactrocera dorsalis) are strongly attracted to a specific phenylpropanoid, methyl eugenol (ME). This leads to voracious consumption of the compound by the male flies, and its subsequent utilization as a sex pheromone precursor (Howlett, 1912; Nishida et al., 1988; Tan and

71 Nishida, 2012). Furthermore, volatiles derived from host fruits play a crucial role in the search for oviposition sites by gravid females. Electrophysiological studies using gas chromatography-flame ionization detection coupled with electroantennographic 
74 detection (GC-EAD) have shown that a number of compounds including terpenes and

75 phenylpropanoids derived from host fruits elicit female antennal responses in $B$.

76 dorsalis (Siderhurst and Jang, 2006a; Siderhurst and Jang, 2006b; Kamala Jayanthi et

77 al., 2012; Kamala Jayanthi et al., 2014; Damodaram et al., 2014).

Although the perception of plant semiochemicals is so important in the

79

80

81

82

83

84

85

86

87

88

89

90

91

92

93

94

95

96

97

98 diverse life history of tephritid fruit flies, as described above, the mechanisms by which chemoreception occurs have not been fully elucidated. The major molecular components of insect chemoreception have been identified mainly from studies on Drosophila melanogaster (Fleischer et al., 2017); chemosensory receptors are essential for the recognition of ligands at peripheral neurons. Insect chemosensory receptors consist of three types of insect-specific superfamilies: olfactory receptors (ORs), gustatory receptors (GRs), and ionotropic receptors (IRs) (Vosshall and Stocker, 2007; Touhara and Vosshall, 2009; Rytz et al., 2013). These receptors are thought to form ligand-gated ion channels and/or to function as G-protein-coupled receptors (GPCRs). Among these insect receptor superfamilies, ORs have been relatively well characterized as heteromeric ligand-gated ion channels that consist of a specific OR and a highly conserved co-receptor ORCO (Sato et al., 2008; Wicher et al., 2008). Because a specific odorous ligand is tuned to a specific OR in this system, the identification of the ligands for uncharacterized ORs could provide clues to the essential chemicals involved in the life cycle of Bactrocera species.

A previous study has shown that the odorant receptor co-receptor, ORCO, is involved in the perception of ME in B. dorsalis, suggesting that specific ORs are required for the chemoreception of ME and its metabolites (Zheng et al., 2012). Furthermore, chemosensory genes that code for ORs, IRs, and GRs have been identified in the transcriptome of B. dorsalis (Wu et al., 2015; Liu et al., 2016). 
99 Importantly, it is possible to access the reference genome sequences of $B$. dorsalis at

100 the National Center for Biotechnology Information (NCBI) website

101 (http://www.ncbi.nlm.nih.gov/genome/10754). Although the sequence data for $B$.

102 dorsalis has been obtained, no chemosensory receptors responding to semiochemicals

103 have been characterized. In the present study, we conducted RNA sequencing (RNA-

104 seq) analysis of the chemosensory organs of $B$. dorsalis to identify genes coding for

105 chemosensory receptors. We identified 60 ORs, 17 GRs, and 23 IRs - including their

106 homologs and variants - from the transcriptome of male antennae and proboscises.

107 We characterized the functional properties of two ORs that respond to plant

108 semiochemicals in a heterologous expression system comprising Xenopus oocytes.

109 We further assessed the attraction of both female and male flies to four volatiles

110 recognized as ligands for the two ORs, and found that when used in combination with

111 visual cues, certain plant volatiles had a significant effect on the landing behavior of

112 mated females. In the present study, we demonstrated the functional identification of

113 specific ligands for chemosensory receptors, which should provide clues to the

114 identity of chemicals that influence insect behaviors.

115

\section{2. Materials and methods}

$118 \quad 2.1$. Insects

119 For preparation of total RNA, we obtained a strain of $B$. dorsalis from a colony

120 maintained by the Naha Plant Protection Station in Okinawa, Japan. The strain-

121 originating in Okinawa, Japan — was reared with the permission of the Minister of

122 Agriculture, Forestry, and Fisheries of Japan (permit No. 56Y-1882). For the

123 behavioral bioassay, we used a laboratory-reared colony of $B$. dorsalis from the 
124 Department of Biology, the Faculty of Science, Universiti Putra Malaysia. The flies

125 were kept at $25-29^{\circ} \mathrm{C}$ and $83-90 \%$ relative humidity, and subjected to a $12-\mathrm{h} \mathrm{light} / 12$ -

$126 \mathrm{~h}$ dark photoperiod regimen. The adult flies were given ad libitum access to water and

127 a mixture of sugar and hydrolyzed protein $(3: 1 \mathrm{w} / \mathrm{w})$. Males and females were

128 separated within 3 days of emergence to prevent mating, and kept in cages $(30 \mathrm{~cm} \times$

$12930 \mathrm{~cm} \times 30 \mathrm{~cm}$ ) until required for the bioassay, which took place $16-19$ days after

130 emergence. Mated flies were obtained in the following manner. Two days before the

131 bioassay, virgin males and females were placed together in a cage in the morning. The

132 mated pairs (>30 min of non-stop copulation) were gently collected in the late

133 evening using a glass vial $(2 \mathrm{~mm}$ diameter $\times 8.5 \mathrm{~cm})$, and placed in a separate cage

134 containing food and water. This was done to ensure that the copulating pairs did not

135 separate from each other. Those flies were then segregated again by sex into separate

136 cages $(40 \mathrm{~cm} \times 40 \mathrm{~cm} \times 40 \mathrm{~cm})$, and allowed to acclimatize in a sheltered outdoor

137 bioassay area that received sunlight from the east before the experiment commenced

138 the next morning. The virgin, males and females were also separated and allowed to

139 acclimatize in the bioassay area prior to the behavioral trials.

141 2.2. RNA sequencing and assembly

142 The male flies were staged at $0-2,2-4,3-5$ and 5-7 days after eclosion.

143 Approximately 150 males were collected from each adult stage. Their antennae and

144 proboscises were dissected and homogenized in TRIzol Reagent (GIBCO-BRL,

145 Gaithersburg, MD, USA). Total RNAs were extracted from the homogenates and

146 purified using NucleoSpin RNA (Macherey-Nagel, Germany). Sequence libraries

147 were prepared using the TruSeq RNA Sample Preparation Kit v2 (Illumina, Inc., San

148 Diego, CA, USA) as described previously (Yang et al., 2015). RNA sequencing was 
149 performed on an Illumina MiSeq system using the MiSeq Reagent Kit v3 600 cycle

150 (Illumina, Inc., San Diego, CA, USA). The reads were preprocessed with

151 Trimmomatic v0.33 (Bolger et al., 2014) for quality trimming using the following

152 parameters: LEADING: 10; TRAILING: 10; SLIDINGWINDOW: 4:20; MINILEN:

153 150. The resulting clean reads data have been deposited in the DNA Data Bank of

154 Japan (DDBJ) Sequence Read Archive under accession number PRJDB6798. The

155 pass-through reads were subjected to de novo assembling using the Trinity, Bowtie,

156 eXpress, and DEGseq (PE) programs (Grabherr et al., 2011) implemented in the

157 maser pipeline of the Cell Innovation Program at the National Institute of Genetics

158 (http://cell-innovation.nig.ac.jp/index en.html). Fragments per kilobase of exon per

159 million (FPKM) values were calculated to estimate the expression levels of the

160 transcripts.

161

162 2.3. Screening and characterization of sequences of candidate chemosensory

163 receptors

164 We identified candidate chemosensory receptor genes from the Trinity contigs using

165 the Pfam database. For this purpose, we obtained four protein domain families of $D$.

166 melanogaster from the Pfam database (http://pfam.xfam.org): $7 \mathrm{tm}$ odorant receptor

167 (PF02949), 7tm chemosensory receptor (PF08395), trehalose receptor (PF06151), and

168 ligand gated ion channel (PF00060). We screened the Trinity contigs by similarity to

169 these protein domain families using a BLASTX search at an Expect value (E-value)

170 threshold of 1e-5. In parallel, we analyzed the Trinity contigs using a TBLASTN

171 search against protein databases consisting of chemosensory receptors of $D$.

172 melanogaster at the same E-value threshold. We obtained open reading frames

173 (ORFs) of the extracted contigs using EMBOSS Transeq 
174 (https://www.ebi.ac.uk/Tools/st/emboss transeq/), and used them as queries in a

175 BLASTP search against the NCBI non-redundant protein database. Contigs that

176 ranked highly with ORs, GRs, or IRs were considered candidate genes coding for

177 insect chemosensory receptors. Overlapping variants with identical ORFs were

178 merged at this step by selecting the longest as the representative transcript of a variant

179 group. Full-length ORFs of several ORs were predicted from genome sequences in

180 the genome sequencing and assembly project, and were cloned into a vector, as

181 described in Section 2.4. Candidate chemosensory receptors were named according to

182 the following criteria. i) Chemosensory receptors were named as described in a

183 previous paper (Wu et al., 2015) when their amino acid sequences were identical. ii)

184 Orthologs of chemosensory receptors uncharacterized from B. dorsalis were named

185 according to those of D. melanogaster. iii) For homologous chemosensory receptors

186 with amino acid similarities of less than $80 \%$, the names of the homologs were

187 differentiated with a numerical postscript, e.g., BdorOR7a-1 and BdorOR7a-2. iv) In

188 cases where the amino acid similarities were $80 \%$ or more, version numbers were

189 assigned to the receptors, e.g., BdorOR67c-v1 and BdorOR67c-v2. v) In cases where

190 multiple partial sequences of a candidate chemosensory receptor were identified, each

191 sequence was labeled -part1, -part2, etc., e.g., BdorOR2a-part1 and BdorOR2a-part2.

192 To compare amino acid sequences of chemosensory receptors identified from $B$.

193 dorsalis between the present study and previous studies, we performed BLASTP

194 searches with an E-value cutoff of 1e-100.

195

197 vector 
198 We cloned full-length coding sequences of candidate ORs into a $\mathrm{pCS} 2 \mathrm{P}+$ vector

199 kindly provided by Prof. Marc Kirschner (https://www.addgene.org/17095/). The

200 primers were designed from the predicted ORFs based on the assembled contigs or

201 reference genome sequences of $B$. dorsalis at the NCBI web site

202 (https://www.ncbi.nlm.nih.gov/genome/?term=JFBF01). The ORFs of BdorORCO,

203 BdorOR94b-1, and BlatOR59a were amplified by PCR from cDNA prepared from

204 male antennae using primers including untranslated regions based on the contig

205 sequences. The PCR products were then cloned into a pGEM-T vector (Promega, WI,

206 USA). The ORFs were modified with a Kozak consensus sequence ( $5^{\prime}$-GCCGCC-

$2073^{\prime}$ ) and the appropriate restriction site by PCR amplification using the following

208 primers. The forward primer included the Kozak consensus sequence followed by a

209 BamHI restriction site, and the reverse primer included an $\mathrm{XbaI}$ restriction site. The

210 PCR products were cloned into a $\mathrm{pCS} 2 \mathrm{P}+$ vector using the restriction sites. The ORFs

211 of the other ORs were amplified by PCR as described above, except that the primers

212 included a part of the pCS2P+ sequence to enable cloning into the vector using an In-

213 Fusion HD cloning Kit (Takara, Otsu, Japan). The PCR reactions were performed

214 using AmpliTaq DNA polymerase (Thermo Fisher Scientific, Waltham, MA, USA)

215 according to the manufacturer's protocol. The primers used for construction are listed

216 in Table S1.

218 2.5. Phylogenetic analysis

219 Deduced amino acid sequences of candidate ORs were aligned using the Clustal W

220 2.1. program (Thompson et al., 1994). Prior to this process, we merged the partial

221 sequences of BdorOR2a, BdorOR7a-8, BdorOR24a, BdorOR45a, and BdorOR63al-

$222 v 1$. We selected candidate ORs with sequences of more than 150 amino acid for 
223 phylogenetic analysis, and constructed a phylogenetic tree from the aligned

224 sequences. We applied the maximum likelihood method with the Jones-Taylor-

225 Thornton (JTT) model with among-site rate heterogeneity according to gamma

226 distribution with invariant sites $(G+I)$ using MEGA5 software (Tamura et al., 2011).

227 We performed 1000 bootstrap replicates.

\subsection{Expression analyses of the candidate receptors by RT-PCR and quantitative}

231 Total RNAs were prepared from various tissues of the staged adults within 2 days of

232 eclosion, as described above. Reverse transcription was performed using the ReverTra

233 Ace qPCR RT Master Mix (TOYOBO, Tsuruga, Japan). The generated cDNAs were

234 subjected to PCR amplification with gene-specific primers using the GoTaq Green

235 Master Mix (Promega, WI, USA). The PCR conditions were: $94^{\circ} \mathrm{C}$ for $1 \mathrm{~min}$; and 35

236 or 40 cycles of $94^{\circ} \mathrm{C}$ for $30 \mathrm{~s}, 60^{\circ} \mathrm{C}$ for $30 \mathrm{~s}, 72^{\circ} \mathrm{C}$ for $1 \mathrm{~min}$, and $72^{\circ} \mathrm{C}$ for $2 \mathrm{~min}$.

237 Alternatively, the cDNA were used as a template for qPCR using the

238 THUNDERBIRD SYBR qPCR Mix (TOYOBO, Tsuruga, Japan) on a Thermal

239 Cycler Dice Real Time System (Takara, Shiga, Japan). We investigated five or six

240 independent biological samples to quantify the levels of transcription. The

241 transcription levels were normalized with $r p S 3$ transcription levels in the same

242 samples. The primers used for RT-PCR and qPCR are listed in Table S2.

\section{2.7. Chemicals}

245 The chemicals used for the functional analysis of the BdorORs are listed in Table S3,

246 and their structures are shown in Fig. S1. We synthesized 3-oxo-7,8-dihydro- $\alpha$-ionone

247 (P3) according to the method described in a previous paper (Enomoto et al., 2010). 
248 We synthesized 4-propionyloxyisophorone (E0P) from 4-oxoisophorone (TCI, Tokyo,

249 Japan) (Nishida and Tan, 2016). Briefly, the carbonyl function of 4-oxoisophorone at

250 C-1 was protected by converting it into a ketal group using ethylene glycol. The

251 carbonyl function at C-4 was then reduced to a hydroxyl moiety, and the ketal at C-1

252 was simultaneously deprotected using $\mathrm{NaBH}_{4}$. The product was then propionylated

253 into E0P using anhydrous propionic acid.

\subsection{Receptor expression in Xenopus oocytes and two-electrode voltage-clamp}

recording

257 The preparation of Xenopus laevis oocytes, the microinjection of receptor gene RNAs,

258 and the recording of whole-cell currents were performed as described previously with 259 minor modifications (Mitsuno et al., 2008). In brief, complementary RNAs (cRNAs)

260 were synthesized from linearized pCS2P+ vectors containing the full-length coding

261 sequences of the ORs using a mMESSAGE mMACHINE T7 Transcription Kit

262 (Thermo Fisher Scientific, Waltham, MA, USA). Stage V to VII Xenopus oocytes

263 treated with collagenase in $\mathrm{Ca}^{2+}$-free saline solution were microinjected with a

264 mixture comprising $O R$ and $B d o r O R C O$ cRNAs (2.5 ng each). Using a two-electrode

265 voltage clamp (OC-725, Warner, Hamden, CT, USA), we recorded whole cell

266 currents from injected oocytes after incubation for 5-7 days at $20^{\circ} \mathrm{C}$ in an assay buffer

267 comprising $96 \mathrm{mM} \mathrm{NaCl}, 2 \mathrm{mM} \mathrm{KCl}, 1.8 \mathrm{mM} \mathrm{CaCl}_{2}, 1.6 \mathrm{mM} \mathrm{MgCl} 2,2.5 \mathrm{mM} 4-(2-$

268 hydroxyethyl)-1-piperazineethanesulfonic acid (HEPES), and $2.5 \mathrm{mM} 2-(N$ -

269 morpholino)ethanesulfonic acid MES ( $\mathrm{pH}$ 7.5). The inward current was monitored at

270 a holding potential of $-80 \mathrm{mV}$. Each ligand was diluted with the assay buffer to a

271 specific concentration containing $0.1 \%$ dimethyl sulfoxide (DMSO). The assay buffer 272 containing $0.1 \%$ DMSO was used as a negative control. Data acquisition and analyses 
273 were carried out using Digidata 1322A and pCLAMP software (Axon Instruments,

274 Foster City, CA, USA).

\subsection{Behavioral bioassays}

277 We evaluated the attraction of the flies to 1-octen-3-ol, geranyl acetate, farnesenes,

278 and linalyl acetate, in combination with white or green sphere models as visual cues.

279 Ethanol was used as a control. We used a total of four spheres (one green with a test

280 compound and one green with ethanol; one white with a test compound and one white

281 with ethanol) for attraction in each of the four groups of flies (Fig. S2A). Sexually

282 mature adult flies (50 virgin females, 50 virgin males, 50 mated females, or 50 mated

283 males) were transferred to a meshed cage $(40 \mathrm{~cm} \times 40 \mathrm{~cm} \times 40 \mathrm{~cm}$; placed in a

284 sheltered outdoor bioassay area) in the morning 1 day prior to commencement of the

285 experiment for acclimatization. On the day of the experiment (08:00-11:00), we

286 impregnated pieces of Whatman ${ }^{\circledR}$ No. 1 filter paper $(15 \mathrm{~mm} \times 3 \mathrm{~mm})$ with $1 \mathrm{mg}$ of

287 each of the test compounds dissolved in $5 \mu \mathrm{L}$ of ethanol, and dried them at room

288 temperature. Each filter paper was then placed in a clean 0.2-mL clear microtube

289 (with the cap removed) (Labchem, Malaysia), which was positioned facing up in one

290 of the holes of a polyethylene sphere that consisted of 26 holes (sphere diameter 40

$291 \mathrm{~mm}$; hole diameter 6 mm; Catalog No. GV0310, Tabata Co., Ltd., Tokyo, Japan).

292 Each sphere was placed on a plastic petri dish (diameter $5 \mathrm{~cm}$ ) to prevent rolling on

293 the cage floor during the bioassay (Fig. S2B). Ethanol was used as a control. Each of

294 the four spheres was then placed $10 \mathrm{~cm}$ from its respective corner in the meshed cage.

295 The position of the spheres based on color and compound combination was re-

296 randomized in each of the 4-6 replicates used, with different cohorts of flies tested 
297 each time. Fruit flies landing on the spheres were counted and rapidly removed by 298 aspiration during the 15-min bioassay.

\subsection{Statistical analysis}

301 Statistical analyses were conducted using R software (www.r-project.org). Dose responses were analyzed using the four-parameter log-logistic model of the $d r c$ extension package (Ritz et al., 2015). For the behavioral bioassay, we used a generalized linear model (GLM) with binomial distribution to determine whether the volatile compound or the color of the sphere significantly affected the number of fruit

306 flies landing on the sphere. The most parsimonious model was identified using the

307 Akaike information criterion (AIC). The likelihood ratio test (LRT) with chi-square 308 distribution was used to determine the difference between the nested models.

\section{Results}

\section{1. $R N A$ sequencing and identification of chemosensory receptors}

313 We obtained 1,675,116 and 2,159,685 raw reads from the transcriptomes of the male

314 antennae and proboscises, respectively, using the Illumina MiSeq system (Table 1).

315 After removing the low-quality, adaptor, and contaminating sequence reads, the male 316 antennae and proboscises yielded 1,159,879 and 1,383,389 clean reads, respectively,

317 which were assembled into 71,766 contigs (S1 text). We identified chemosensory 318 receptors - namely, ORs, GRs, and IRs - by a BLASTX search of the contigs against 319 amino acid sequences and Pfam domains of chemosensory receptors in $D$.

320 melanogaster (Table 2). 
A homology search based on the Pfam domains of the $7 \mathrm{tm}$ odorant receptor

322 (PF02949) and the amino acid sequences of the Drosophila ORs revealed 60

323 candidate ORs. The full-length coding sequences of 13 ORs were expected by de

324 novo assembly. We also used a BLASTN search to predict the full-length coding

325 sequences of three ORs-BdorOR13a, BdorOR63a-2-v1, and BdorOR67c-v1 - and

326 determined their sequences by RT-PCR. Interestingly, we found multiple homologous

327 genes for several ORs including BdorOR7a and BdorOR67d (Table 2), whereas the

328 corresponding Drosophila ORs have only one gene. The divergence of the BdorOR7a

329 subfamily in the phylogenetic tree is remarkable (Fig. 1).

330 We identified four GRs that are homologous to sugar receptors such as the

331 GR5a and GR64 subfamilies by a homology search based on the Pfam domains of

332 trehalose receptors (PF06151) (Freeman and Dahanukar, 2015). We identified another

33313 GRs using the Pfam domains of $7 \mathrm{tm}$ chemosensory receptors (PF08395). We

334 identified two GRs as BdorGR21a and BdorGR63a, which are carbon dioxide

335 receptors and are highly conserved in insect species (Jones et al., 2007; Kwon et al.,

336 2007). We also found four homologous genes of BdorGR21a. Apart from the

337 trehalose and carbon dioxide receptors, we identified eight GRs from the Trinity

338 contigs, one of which was a homolog of the fructose receptor GR43a (Sato et al., 339 2011).

340 We identified ligand-gated ion channels by a homology search based on the

341 Pfam domains of ligand-gated ion channels (PF00060) and Drosophila IRs. Of those,

342 we identified the candidate IRs by a BLASTP search based on translated protein

343 sequences. Among the IRs, we identified two - BdorIR8 a and BdorIR25a - as

344 ionotropic co-receptors (Benton et al., 2009; Abuin et al., 2011). We also found a 
345 homologous gene of BdorIR8a, and a pair of homologous genes in BdorIR31a,

346 BdorIR64a, BdorIR75a, BdorIR76a, BdorIR92a, and BdorIR93a.

Previous studies have identified candidate chemosensory receptors in $B$.

348 dorsalis (Wu et al., 2015; Liu et al., 2016). A comparison of those receptors with the

349 chemosensory receptors found in the present study indicated the novel ORs, GRs, and

350 IRs listed in Table 2. The coding sequences of the candidate chemosensory receptor

351 genes and their accession numbers are shown in the S2 text and Table S4,

352 respectively.

\subsection{Expression profiles of the ORs}

Sex-specific behaviors-including attraction and oviposition responses to

356 plant semiochemicals - have been observed in B. dorsalis (Howlett, 1912; Nishida et

357 al., 1988; Siderhurst and Jang, 2006a; Siderhurst and Jang, 2006b; Kamala Jayanthi et

358 al., 2012; Kamala Jayanthi et al., 2014; Damodaram et al., 2014). Therefore, we used

359 qPCR to compare the transcription levels of 15 ORs with known full-length coding

360 sequences in female and male antennae to determine if expression was sex-specific.

361 All the ORs tested were expressed in both sexes, although we did observe a

362 significant difference in the transcription level of BdorOR35a between the female and

363 male antennae (Fig. 2).

The expression of candidate chemosensory receptors in the male antenna and

365 proboscises was predicted by FPKM analysis (Table S5). Whereas most of the

366 transcripts coding ORs had various FPKM values in the antennae, some including

367 BdorORCO also had relatively low FPKM values in the proboscises. With regard to

368 the GRs, the transcripts coding carbon dioxide receptors-namely BdorGR21a and its

369 variants - had high FPKM values in the antennae. Some of the transcripts coding 
370 GRs — including the sugar receptor subfamilies BdorGR5a and BdorGR64 — only had

371 high FPKM values in the proboscises, whereas the transcripts coding BdorGR28b and

372 BdorGR8a had FPKM values in the antennae, but not in the proboscises. Most of the

373 transcripts coding IRs had various FPKM values in the antennae, and some had

374 FPKM values in the proboscises. In contrast, the transcripts coding BdorIR56c and

375 BdorIR93a-2 only had FPKM values in the proboscises.

We used RT-PCR to examine the transcription profile of $B d o r O R C O$ in

various tissues including olfactory and gustatory organs (Fig. 3A). BdorORCO was

expressed primarily in the olfactory organs, antennae, and maxillary palps, although

we observed marginal expression in the proboscises. We used qPCR to quantitatively

380 compare the transcription levels of this gene in female and male antennae,

381 proboscises, and tarsi (Fig. 3B). BdorORCO was highly expressed in both female and

382 male antennae in accordance with its role as an obligatory co-receptor. In contrast, the

383 transcription levels of $B d o r O R C O$ were extremely low in both female and male

384 proboscises (less than one thousandth of those in the antennae). The transcription

385 levels of BdorORCO in both female and male tarsi were as low as one tenth those in

386 the proboscises.

We also used RT-PCR to analyze the expression profiles of the selected ORs

$388 B d o r O R 13 a$ and BdorOR82a (Fig. 3A). The PCR products of these genes were

389 detected by 40 cycles of amplification, but not by 35 cycles, probably owing to the

390 low transcription levels. We observed BdorOR $13 a$ expression in both female and

391 male antennae, and in male maxillary palps and gustatory organs, i.e., the proboscises

392 and foreleg tarsi. We observed BdorOR82a expression in both female and male

393 antennae, and in male maxillary palps and female foreleg tarsi. We used qPCR to

394 compare transcription levels in females and males to determine if BdorOR13a and 
395 BdorOR82a were expressed in a sexually dimorphic pattern. There were extremely

396 low BdorOR13a and BdorOR82a transcription levels in both the proboscises and

397 foreleg tarsi (Fig. 3C). There were no significant differences in the transcription levels

398 of these genes between female and male proboscises or foreleg tarsi $(p>0.05$,

399 Student's $t$-test).

400

401

3.3. Identification of ligands for BdorOR13a and BdorOR82a by two-electrode

402

voltage-clamp recording

403 To identify the ligands for the ORs in B. dorsalis, we co-expressed each of the ten

404 receptor proteins-BdorOR7a-4, BdorOR7a-7, BdorOR13a, BdorOR35a,

405 BdorOR43a-2-v1, BdorOR63a-2-v1, BdorOR67c-v1, BdorOR67d-1, BdorOR74a and

406 BdorOR82a - with the obligatory co-receptor BdorORCO in Xenopus oocytes. We

407 tested 24 compounds including host plant chemicals, male attractants, and sex

408 pheromones for Bactrocera species, as shown in Table S3 and Fig. S1. Of the ten

409 receptor proteins tested, BdorOR13a responded to one compound and BdorOR82a

410 responded to three compounds. The oocyte co-expressing BdorOR13a with

411 BdorORCO responded significantly to 1-octen-3-ol at a concentration of $100 \mu \mathrm{M}$

412 (Fig. 4A-C). The current value induced by 1-octen-3-ol was significantly higher than

413 that induced by the control (DMSO). The current induced by 1-octen-3-ol increased in

414 a dose-dependent manner, and the $\mathrm{EC}_{50}$ value was $52.0 \mu \mathrm{M}$ (Fig. 4D, E). The oocyte

415 co-expressing BdorOR82a and BdorORCO responded significantly to geranyl acetate,

416 and responded weakly to farnesenes and linalyl acetate at a concentration of $100 \mu \mathrm{M}$

417 (Fig. 5A-C). Further experiments revealed significant differences between the current

418 values of these compounds and that of the control (Fig. 5D). The current induced by 
419 geranyl acetate increased in accordance with an increase in concentration, but we did

420 not observe a plateau at the maximum concentration tested (Fig. 5E, F).

421 We compared the amino acid sequences of the characterized ORs - BdorOR13a and

422 BdorOR82a - with those of the Drosophila ORs because the properties of these ORs

423 have been well characterized in D. melanogaster. BLASTP analysis indicated that the

424 deduced amino acid sequences of BdorOR13a and BdorOR82a were similar to the 425 sequences of DmOR13a (GenBank: AAF48549.2) and DmOR82a (GenBank:

426 AAN13335.1), with 51\% and 43\% amino acid identities, respectively. Alignments of 427 these ORs revealed that the amino acids within the transmembrane domains are well 428 conserved (Fig. S3).

\subsection{Behavioral bioassay}

431 We examined the effect of volatiles characterized as ligands for BdorOR13a and

432 BdorOR82a on the landing behavior of $B$. dorsalis. To determine whether there were

433 behavioral differences between the sexes in terms of mating, we tested both females

434 and males individually with four volatiles - 1-octen-3-ol, geranyl acetate, farnesenes, 435 and linalyl acetate - before and after mating. Because hardly any flies were attracted 436 to the volatile-treated filter papers when used alone, we placed two sets of green and 437 white spheres in the cage as visual cues, each containing microtubes with filter papers

438 treated with or without the aforementioned volatiles (Fig. S2). We attempted to

439 determine whether volatiles or color affected the number of flies landing on each

440 sphere using the GLM model. When the fruit flies were exposed to 1-octen-3-ol- the

441 ligand for BdorOR13a - the volatile factor but not the color factor alone significantly

442 affected the numbers of mated females, and the numbers of both virgin and mated

443 males landing on the spheres (Table 3). The number of mated females landing on the 
444 spheres increased by the exposure to 1-octen-3-ol (Fig. 6A). Furthermore, we

445 observed a few of the mated females probing the surface of the sphere with abdominal

446 bending and aculeus extension, which are typical oviposition behaviors. However, the

447 numbers of both virgin and mated males decreased by the exposure to 1-octen-3-ol

448 (Fig. 6A). When the fruit flies were exposed to geranyl acetate or farnesenes - the

449 ligands for BdorOR82a - the volatile factor but not the color factor alone significantly

450 affected the behavior of the mated females, and more of them landed on the spheres

451 emitting the volatiles (Table 3, Fig. 6A). In contrast, the number of mated males

452 landing on the spheres decreased by the exposure to farnesenes. When the fruit flies

453 were exposed to linalyl acetate, the volatile factor did not affect the number of fruit

454 flies landing on the spheres, but color alone significantly increased the number of

455 mated females (Table 3). Taken together, these results indicate that exposure to each

456 of the volatiles - namely 1-octen-3-ol, geranyl acetate and farnesenes - significantly

457 affected the landing behavior of the mated $B$. dorsalis females, whereas the males

458 seemed to avoid the spheres that emitted 1-octen-3-ol or farnesenes. We confirmed

459 that exposure to ethanol as a solvent did not affect the landing behavior of the mated

460 females (Fig. S4A). Although the total number of mated females landing on the four

461 spheres emitting 1-octen-3-ol, geranyl acetate, or farnesenes was significantly higher

462 than in the other groups (Fig. 6B), there was no significant difference in the number

463 of females landing on the spheres when the volatile-emitting and ethanol-emitting

464 spheres (the control) were compared (Fig. S4B). This suggests that mated females

465 frequently landed on spheres regardless of whether they were emitting a volatile.

466

467 4. Discussion

468 4.1. Repertoires of chemosensory receptor families in B. dorsalis 
469 We identified multiple candidate chemosensory receptors in B. dorsalis-including 470 novel ORs, GRs, and IRs not reported in previous studies - by transcriptome analysis.

471 We found divergent homologs and variants in several ORs from B. dorsalis,

472 suggesting that ancient genes have diverged by gene duplication in these OR families

473 during adaptation to environmental odorants such as plant volatiles. The physiological

474 roles of the highly divergent BdorOR7a family are of particular interest because $B$.

475 dorsalis seems to require homologous ORs to detect specific odorants or sets of

476 similar odorants. With regard to GRs, we identified two highly conserved carbon

477 dioxide receptors, sugar receptors, and several other receptors. We identified 23 IRs

478 including the homologs of Drosophila ionotropic co-receptors IR8a and IR25a. We

479 found several IRs with two variants - e.g., BdorIR75a, BdorIR76a and BdorIR93a-

480 suggesting that gene duplication has occurred in these IR families, as in the ORs. It is

481 interesting to note that Drosophila OR67d and GR32a have been characterized as

482 receptors for volatile and contact pheromones, respectively (Kohl et al., 2015).

483 OR67d functions as a receptor for 11-cis-vaccenyl acetate (Ha, 2006; Kurtovic et al.,

484 2007); this compound acts as an anti-aphrodisiac pheromone in males to avoid male-

485 male courtship (Zawistowski and Richmond, 1986), but also acts as an aggregation

486 signal in both sexes (Bartelt et al., 1985). The aggregation behavior of males-known

487 as lek formation - has been observed in B. dorsalis and related species (Iwahashi and

488 Majima, 1986; Tan and Nishida, 1996). Therefore, it would be intriguing if the

489 divergent receptors (i.e., members of the BdorOR67d family) were involved in such 490 social behavior.

491

492 4.2. Expression profiles of chemosensory receptors of $\mathrm{B}$. dorsalis 
493 In Drosophila, OR genes are expressed exclusively in olfactory organs (Vosshall et

494 al., 2000), and GR genes are mainly expressed in gustatory organs, with some

495 exceptions such as the expression of GR21a, GR63a, and GR22e in antennae (Scott et

496 al., 2001; Dunipace et al., 2001; Thorne and Amrein, 2008). In contrast, we acquired

497 sequences coding multiple ORs from reads derived from the proboscises, although the

498 FPKM values of these transcripts were low. Likewise, we found transcripts of several

499 GR genes in the sequencing reads from the antennae. Among these, BdorGR8a and

$500 B d o r G R 28 b$ had relatively high FPKM values in the antennae, but no FPKM values in

501 the proboscises. Therefore, differences in the expression profiles of several OR and

502 GR genes in the olfactory and gustatory organs are more obscure in $B$. dorsalis than in

503 D. melanogaster. Because the males of many Bactrocera species are strongly

504 attracted to specific compounds - e.g., B. dorsalis to ME—and subsequently feed

505 voraciously on the compounds, the perception of attractants probably involves both

506 olfactory and gustatory stimulation. A previous study demonstrated that ORCO is

507 required for the attraction of $B$. dorsalis to ME (Zheng et al., 2012), suggesting the

508 involvement of ORs in ME reception. It is also possible that IRs mediate the detection

509 of male attractants in B. dorsalis, because IRs function as both olfactory and gustatory

510 receptors (Rytz et al., 2013; Fleischer et al., 2017). In the present study, although we

511 found most transcripts of BdorIRs in sequencing reads from the antennae, we found

512 others in reads from both antennae and proboscises, or from proboscises only. The

513 question of whether ORs, GRs, IRs, or some combination of them is required for the

514 chemoreception of the attractants is very interesting.

515 Although sexually dimorphic behavior in response to semiochemicals has been

516 reported in B. dorsalis (Howlett, 1912; Nishida et al., 1988; Tan and Nishida, 2012),

517 in the present study we found no distinction between females and males in terms of 
518 the transcription levels of almost all the ORs tested. Conversely, in lepidopteran

519 species there is sex-specific expression of the chemosensory receptors required for

520 sexually dimorphic behavior such as mating or oviposition in chemosensory organs.

521 Since the characterization of a sex pheromone receptor from the silkworm Bombyx

522 mori (Sakurai et al., 2004; Nakagawa et al., 2005), lepidopteran receptors that

523 perceive female pheromones, all of which are specifically expressed in male antennae,

524 have been identified in various genera including Plutella, Mythimna, Diaphania,

525 Antheraea, and Ostrinia (Mitsuno et al., 2008; Forstner et al., 2009; Miura et al.,

526 2009; Miura et al., 2010; Wanner et al., 2010). Other than sex pheromone receptors,

527 the female-specific expression of lepidopteran gustatory receptor for the detection of

528 an oviposition stimulant contained in host plant leaves has been reported in the

529 swallowtail butterfly Papilio xuthus (Ozaki et al., 2011). These findings suggest that

530 the sex-specific expression of chemosensory receptors is closely related to sexually

531 dimorphic behavior in lepidopteran species. In D. melanogaster, however,

532 chemosensory receptor genes are mostly expressed in both sexes, whereas the

533 gustatory pheromone receptor GR68a is specifically expressed in male taste neurons

534 in the foreleg tarsi (Bray and Amrein, 2003). Therefore, the chemosensory

535 information involved in sexually dimorphic behavior triggered by semiochemicals is

536 processed by the central nervous system, rather than the peripheral, in dipteran species

537 including $B$. dorsalis.

538

539 4.3. Binding properties of ORs that respond to plant volatiles

540 In the present study, we characterized two ORs that respond to plant volatiles using

541 Xenopus oocytes as a heterologous expression system. BdorOR13a responded

542 strongly to 1-octen-3-ol, as reported in its homologs in D. melanogaster and the 
543 mosquito species Anopheles gambiae and Aedes aegypti (Lu et al., 2007; Kreher et

544 al., 2008; Bohbot and Dickens, 2009). BdorOR82a responded to geranyl acetate, as

545 reported in its homologs in D. melanogaster and A. gambiae (Hallem et al., 2004;

546 Wang et al., 2010). BdorOR82a also responded significantly to farnesenes and linalyl

547 acetate, whereas a response to these compounds by the homologous OR in $D$.

548 melanogaster has not been reported, to the best of our knowledge. Whereas the

549 response of BdorOR13a to 1-octen-3-ol reached a plateau at an approximate

550 concentration of $100 \mu \mathrm{M}$, the response of BdorOR82a to geranyl acetate failed to

551 reach a plateau up to $10 \mathrm{mM}$, the maximum concentration tested. Furthermore,

552 BdorOR82a responded weakly to farnesenes and linalyl acetate at $100 \mu \mathrm{M}$. Whereas

553 1-octen-3-ol contains a hydroxyl group, geranyl acetate, the isomers of farnesenes and

554 linalyl acetate lack hydrophilic groups, suggesting that the latter three compounds are

555 relatively insoluble in buffer solutions owing to their hydrophobicity. The sparing

556 solubility of geranyl acetate in buffer solution may explain the weak response of

557 BdorOR82a, even at $1 \mathrm{mM}$. Generally, odorant-binding proteins allow hydrophobic

558 ligands to access the receptor neurons of insect chemosensilla, which are surrounded

559 by an aqueous lymphatic fluid (Fleischer et al., 2017). Therefore, geranyl acetate,

560 farnesenes, and linalyl acetate could effectively access BdorOR82a on receptor

561 neurons mediated by binding proteins in vivo. An alternative explanation for the weak

562 response is the low affinity of BdorOR82a for geranyl acetate. Because the amino

563 acid identity between BdorOR82a and DmOR82a is not necessarily high, differences

564 in amino acid residues may affect the sensitivity and specificity of the heteromeric

565 insect OR82a/ORCO complex.

566 BdorOR82a responded to the three hydrophobic compounds. Geranyl acetate

567 and linalyl acetate are both isomeric monoterpenes, but linalyl acetate is branched in a 
568 different way. Farnesenes are sesquiterpene hydrocarbons that differ from geranyl

569 acetate and linalyl acetate in both size and nature. Of the compounds that have been

570 tested, the D. melanogaster DmOR82a homolog responds exclusively to geranyl

571 acetate (Hallem et al., 2004; Hallem and Carlson, 2006), but it is unclear whether

572 DmOR82a also responds to linalyl acetate and farnesenes. Although BdorOR82a has

573 a low E-value (4e-98) against DmOR82a according to a BLASTP search, the amino

574 acid sequences of BdorOR82a and DmOR82a have only $43 \%$ identity. Therefore, it

575 would be interesting if DmOR82a responds to linalyl acetate and farnesenes as does

576 BdorOR82a. A comparison of the binding affinities and amino acid sequences of

577 BdorOR82a and DmOR82a would provide information about the specificities of these

578 receptors.

579

\subsection{Biological function of volatiles characterized as ligands for BdorORs}

581 We found that three ligands for BdorORs - namely, 1-octen-3-ol, geranyl acetate and

582 farnesenes - significantly affect the landing behavior of adult flies. The results

583 suggest that adult flies respond to plant volatiles via the ORs characterized in the

584 present study. However, it should be noted that at least one other OR may be involved

585 in information processing at the peripheral and/or central nervous system level during

586 the response to these volatiles.

587 Interestingly, the ligands had different effects depending on the sex and

588 mating status of the flies. For example, 1-octen-3-ol increased the number of mated

589 females landing on the spheres but reduced the numbers of virgin and mated males

590 landing on the spheres. It should be noted that 1-octen-3-ol has been identified as an

591 oviposition stimulant of $B$. dorsalis in studies on the volatile components of a host

592 fruit (mango; Mangifera indica); gravid females laid more eggs on discs treated with 
593 1-octen-3-ol in binary choice tests (Kamala Jayanthi et al., 2014). We also found that

594 a few of the mated females exhibited oviposition behavior on the surfaces of the

595 spheres emitting 1-octen-3-ol. Our findings suggest that in tephritids, mating causes a

596 switch from normal to oviposition-related behavior, as observed in B. dorsalis. A

597 study on female Mediterranean fruit flies (Ceratitis capitata) has also demonstrated

598 that mating confers a preferential switch; mated females choose host fruit odor over 599 male pheromones (Jang, 1995). The negative effects on the landing behavior of males,

600 regardless of mating experience, suggest that information processing after the

601 perception of volatiles differs between the sexes. It is worth noting that various

602 dipteran behavioral responses to 1-octen-3-ol have been reported, e.g., its ability to

603 attract D. melanogaster larvae (Kreher et al., 2008), and its ability to repel adult

604 females of the spot wing drosophila D. suzukii (Wallingford et al., 2016). Anopheles

605 gambiae and Aedes aegypti mosquitoes are attracted to 1-octen-3-ol in the breath of

606 animals (Kline, 1994). It would be interesting to know if OR13a homologs are

607 associated with these different behaviors in dipteran species, or if another receptor is

608 involved.

Geranyl acetate, farnesenes, and linalyl acetate, which are ligands for

610 BdorOR82a, have been detected in the tropical almond fruit Terminalia catappa, one

611 of the hosts of B. dorsalis (Siderhurst and Jang, 2006b); geranyl acetate and methyl

612 eugenol elicited the largest electroantennogram detection (EAD) responses from the

613 antennae of $B$. dorsalis among the volatiles collected from T. catappa (Siderhurst and

614 Jang, 2006b). Linalyl acetate and the isomers of farnesenes also elicited EAD

615 responses to some extent (Siderhurst and Jang, 2006b). In accordance with the EAD

616 responses, exposure to geranyl acetate or farnesenes had a significant effect on the

617 landing behavior of $B$. dorsalis in our experiments, and we observed different 
618 responses to 1-octen-3-ol with regard to gender and mating experience. These results 619 suggest that the electrophysiological responses to geranyl acetate and farnesenes are 620 linked to their effects on the landing behavior of fruit flies.

621 Geranyl acetate also seems to be important for D. melanogaster, because

622 DmOR82a responds to its analog geranyl acetone, probably as a signal for fruit 623 ripening (Mansourian and Stensmyr, 2015). It would be interesting to see if OR82a

624 homologs, which respond to geranyl acetate and its analogs, commonly mediate 625 semiochemical information released from host fruits in fruit flies of the families 626 Drosophilidae and Tephritidae. Recently, ORs that respond to geranyl acetate and an 627 isomer of farnesene have been identified in aphid and moth species (Liu et al., 2014; 628 Zhang et al., 2017), although these receptors do not belong to the OR82a family. 629 ApisOR5 from the aphid Acyrthosiphon pisum responds to the alarm pheromone (E)$630 \quad \beta$-farnesene and the repellent geranyl acetate, even though there is no homology 631 between ApisOR5 and OR82a at the amino acid level. Instead, ApisOR5 most closely 632 resembles OR85f, with an E-value of 1.6e-4 according to a BLASTP search against 633 Drosophila ORs. Similarly, SexiOR3, which has been identified in the beet

634 armyworm moth Spodoptera exigua, responds to (E)- $\beta$-farnesene, but most closely 635 resembles OR13a, with an E-value of 7.9e-6 according to a BLASTP search against 636 Drosophila ORs. The relationships between the binding properties and structures of 637 these ORs, which share common ligands, are intriguing.

638 To the best of our knowledge, the present study was the first attempt to functionally 639 characterize the ORs of tephritid fruit flies using Xenopus oocytes as a heterologous 640 expression system. The results will enable us to further characterize the orphan 641 receptors of Tephritidae. Furthermore, the identification of ligands for chemosensory 642 receptors will provide information about the important chemicals that affect the life 
643 cycles of fruit flies. Despite the characterization of the BdorORs, we have not

644 provided direct evidence to link the properties of ORs with behavior as an output of

645 signal processing mediated by these receptors. Insect genome engineering using the

646 CRISPR/Cas9 system is now available for Bactrocera species (Choo et al., 2017), and

647 the loss of the function of specific chemosensory receptors of interests will clarify

648 their roles in vivo. This will lead to the elucidation of the mechanisms underlying the

649 chemoreception of various semiochemicals, including plant volatiles, male-specific

650 attractants, and sex pheromones.

651

\section{Acknowledgements}

653 We thank Isao Miyazaki, Hiroyuki Adachi and Takuji Yamamoto of Naha Plant

654 Protection Station (Okinawa, Japan) for providing the fruit flies. We also thank

655 Shinya Kanno, Yurie Hirosaki, Farhana Ainie, and Nor Nasuha for technical

656 assistance. HO was supported by JSPS KAKENHI Grant Number 26450466. 


\section{$658 \quad$ Figure captions}

659

660 Fig. 1. Phylogenetic tree of candidate olfactory receptors (ORs) identified in

661 Bactrocera dorsalis. Branch length is proportional to genetic distance estimated by

662 the maximum likelihood method. The values shown at the nodes of the branches are

663 bootstrap values (\%) from 1000 replicate samplings. The numbers in parentheses

664 indicate the numbers of amino acids in the ORs. "F" or "P" in parentheses indicate

665 full or partial determination of the coding sequence of an OR, respectively. ORs

666 expressed in Xenopus oocytes are indicated in bold.

667

668 Fig. 2. Transcription levels of olfactory receptors (ORs) identified in Bactrocera

669 dorsalis. FA: female antennae; MA: male antennae. Each value is plotted as a dot $(n=$

670 6). The box plot shows $25-75 \%$ (box), median (band inside), and minima to maxima

671 (whiskers). Student's $t$-test: ${ }^{*} p<0.05$.

672

673 Fig. 3. Expression patterns of olfactory receptors (ORs) identified in Bactrocera

674 dorsalis. (A) Expression of ORs in various tissues detected by RT-PCR. PCR

675 amplifications were performed in 35 cycles for $B d o r O R C O$ and $r p S 3$, and 40 cycles

676 for BdorOR13a and BdorOR82a. The lanes are as follows: AT: antennae; MP:

677 maxillary palps; PB: proboscises; FT: foreleg tarsi; ML: midlegs; HL: hindlegs. (B)

678 Transcription levels of $B d o r O R C O$ in chemosensory organs. (C) Transcription levels

679 of BdorOR13a and BdorOR82a in gustatory organs. (B, C) FA: female antennae; MA:

680 male antennae; FP: female proboscises; MP: male proboscises; FT: female tarsi; MT:

681 male tarsi. Each value is plotted as a dot $(n=5-6)$. The box plot shows $25-75 \%$

682 (box), median (band inside), and minima to maxima (whiskers). 
684 Fig. 4. Responses of Xenopus oocytes expressing BdorOR13a with BdorORCO to 685 various compounds. (A) Current trace of an oocyte upon successive exposures to 25 686 samples including DMSO (the control). Each chemical was applied at the time 687 indicated by the arrowhead. (B) Currents measured in the oocytes. The structure of 688 each compound and its corresponding abbreviation is shown in Fig. S1. The number 689 in parentheses after each compound corresponds to the number on the arrowhead in 690 (A). Error bars indicate SE $(n=3)$. Student's $t$-test: $* p<0.05$. (C) Structure of a 691 ligand for BdorOR13a. (D) Responses of an oocyte expressing BdorOR13a with 692 BdorORCO to 1-octen-3-ol at various concentrations. (E) Dose-response curve of 693 oocytes responding to 1-octen-3-ol. Each point represents the mean current value. 694 Error bars indicate SE $(n=9-11)$.

695

696 Fig. 5. Responses of Xenopus oocytes expressing BdorOR82a with BdorORCO to 697 various compounds. (A) Current trace of an oocyte upon successive exposures to 25 698 samples including DMSO (the control). Each chemical was applied at the time 699 indicated by the arrowhead. (B) Currents measured in the oocytes. The structure of 700 each compound and its corresponding abbreviation is shown in Fig. S1. The number 701 in parentheses after each compound corresponds to those on the arrowheads in (A).

702 Error bars indicate SE $(n=3)$. Student's $t$-test: * $p<0.05$. (C) Structures of ligands for 703 BdorOR82a. The structure of one of the farnesene isomers is shown. (D) Currents 704 measured in oocytes responding to L-OAc (linalyl acetate), FRN-mix (a mixture of

705 farnesene isomers), and G-OAc (geranyl acetate) at $100 \mu \mathrm{M}$. Each value is plotted as 706 a dot $(n=9-10)$. The box plot shows 25-75\% (box), median (band inside), and 707 minima to maxima (whiskers). Student's $t$-test: ${ }^{*} p<0.05,{ }^{*} p<0.01$. (E) Responses 
708 of an oocyte expressing BdorOR82a with BdorORCO to geranyl acetate at various

709 concentrations. (F) Dose-response curve of oocytes responding to geranyl acetate.

710 Each point represents the mean current value. Error bars indicate SE $(n=8-9)$.

711

712 Fig. 6. Effects of volatiles and visual cues on landing behaviors of Bactrocera

713 dorsalis. The box plot shows $25-75 \%$ (box), median (band inside), and minima to

714 maxima (whiskers). Virgin-F, Mated-F, Virgin-M, and Mated-M indicate virgin

715 females, mated females, virgin males, and mated males, respectively. (A) Numbers of

716 virgin or mated females or males landing on green or white spheres. The numbers of

717 flies are plotted as dots $(n=5-6)$. Significant effects of volatiles or colors indicated in

718 Table 3 are shown in the categories of fruit flies as $\mathrm{V}$ or $\mathrm{C}$ with $p$-values $(<0.05)$,

719 respectively. T-G, T-W, C-G, and C-W indicate volatile-treated green balls, volatile-

$\mathbf{7 2 0}$ treated white balls, control (volatile-untreated) green balls, and control white balls,

721 respectively. (B) Total numbers of fruit flies landing on the spheres calculated from

722 (A). Boxes with letters are significantly different at $p<0.05$ according to Tukey's

$\mathbf{7 2 3}$ HSD test. 
References

Abuin, L., Bargeton, B., Ulbrich, M.H., Isacoff, E.Y., Kellenberger, S., Benton, R., 2011. Functional architecture of olfactory ionotropic glutamate receptors. Neuron 69, 44-60. doi:10.1016/j.neuron.2010.11.042

Bartelt, R.J., Schaner, A.M., Jackson, L.L., 1985. cis-Vaccenyl acetate as an aggregation pheromone in Drosophila melanogaster. J. Chem. Ecol. 11, 1747-1756. doi:10.1007/BF01012124

Benton, R., Vannice, K.S., Gomez-Diaz, C., Vosshall, L.B., 2009. Variant ionotropic glutamate receptors as chemosensory receptors in Drosophila. Cell 136, 149-162. doi:10.1016/j.cell.2008.12.001

Bohbot, J.D., Dickens, J.C., 2009. Characterization of an enantioselective odorant receptor in the yellow fever mosquito Aedes aegypti. PLoS One 4(9), e7032. doi:10.1371/journal.pone.0007032

Bolger, A.M., Lohse, M., Usadel, B., 2014. Trimmomatic : a flexible trimmer for Illumina sequence data. Bioinformatics 30, 2114-2120. doi:10.1093/bioinformatics/btu170

Bray, S., Amrein, H., 2003. A putative Drosophila pheromone receptor expressed in male-specific taste neurons is required for efficient courtship. Neuron 39, 1019-1029. doi:10.1016/S0896-6273(03)00542-7

Choo, A., Crisp, P., Saint, R., O'Keefe, L.V., Baxter, S.W., 2017. CRISPR/Cas9mediated mutagenesis of the white gene in the tephritid pest Bactrocera tryoni. J. Appl. Entomol. 142, 1-7. doi:10.1111/jen.12411

Damodaram, K.J.P., Kempraj, V., Aurade, R.M., Venkataramanappa, R.K., Nandagopal, B., Verghese, A., Bruce, T., 2014. Oviposition site-selection by Bactrocera dorsalis is mediated through an innate recognition template tuned to $\gamma$-octalactone. PLoS One 9(1), e85764. doi:10.1371/journal.pone.0085764

Dunipace, L., Meister, S., McNealy, C., Amrein, H., 2001. Spatially restricted expression of candidate taste receptors in the Drosophila gustatory system. Curr. Biol. 11, 822-835. doi:10.1016/S0960-9822(01)00258-5

Enomoto, H., Ishida, T., Hamagami, A., Nishida, R., 2010. 3-Oxygenated $\alpha$ ionone derivatives as potent male attractants for the solanaceous fruit fly, 
758

759

760

Bactrocera latifrons (Diptera: Tephritidae), and sequestered metabolites in the rectal gland. Appl. Entomol. Zool. 45, 551-556. doi:10.1303/aez.2010.551

Fleischer, J., Pregitzer, P., Breer, H., Krieger, J., 2017. Access to the odor world: olfactory receptors and their role for signal transduction in insects. Cell. Mol. Life Sci. 1-24. doi:10.1007/s00018-017-2627-5

Forstner, M., Breer, H., Krieger, J., 2009. A receptor and binding protein interplay in the detection of a distinct pheromone component in the silkmoth Antheraea polyphemus. Int. J. Biol. Sci. 5, 745-757.

Freeman, E.G., Dahanukar, A., 2015. Molecular neurobiology of Drosophila taste. Curr. Opin. Neurobiol. 34, 140-148. doi:10.1016/j.conb.2015.06.001

Grabherr, M.G., Haas, B.J., Yassour, M., Levin, J.Z., Thompson, D.A., Amit, I., Adiconis, X., Fan, L., Raychowdhury, R., Zeng, Q., Chen, Z., Mauceli, E., Hacohen, N., Gnirke, A., Rhind, N., Di Palma, F., Birren, B.W., Nusbaum, C., Lindblad-Toh, K., Friedman, N., Regev, A., 2011. Full-length transcriptome assembly from RNA-Seq data without a reference genome. Nat. Biotechnol. 29, 644-652. doi:10.1038/nbt.1883

Ha, T.S., Smith, D.P., 2006. A pheromone receptor mediates 11-cis-vaccenyl acetate-induced responses in Drosophila. J. Neurosci. 26, 8727-8733. doi:10.1523/JNEUROSCI.0876-06.2006

Hallem, E.A., Carlson, J.R., 2006. Coding of odors by a receptor repertoire. Cell 125, 143-160. doi:10.1016/j.cell.2006.01.050

Hallem, E.A, Ho, M.G., Carlson, J.R., 2004. The molecular basis of odor coding in the Drosophila antenna. Cell 117, 965-79. doi:10.1016/j.cell.2004.05.012

Howlett, F., 1912. Chemical reactions of fruit-flies. Bull. Entomol. Res. 6, 297305. doi:10.1017/S0007485300000328

Iwahashi, O., Majima, T., 1986. Lek formation and male-male competition in the melon fly, Dacus cucurbitae Coquillett (Diptera : Tephritidae). Appl. Entomol. Zool. 21, 70-75. doi:http://doi.org/10.1303/aez.21.70

Jang, E.B., 1995. Effects of mating and accessory gland injections on olfactorymediated behavior in the female Mediterranean fruit fly, Ceratitis capitata. J. Insect Physiol. 41, 705-710. doi:10.1016/0022-1910(95)00015-M

Jones, W.D., Cayirlioglu, P., Kadow, I.G., Vosshall, L.B., 2007. Two chemosensory receptors together mediate carbon dioxide detection in Drosophila. Nature 445, 86-90. doi:10.1038/nature05466 
792

793

794

795

796

797

798

799

800

801

802

803

804

805

806

807

808

809

810

811

812

813

814

815

816

817

818

819

820

821

822

823

824

Kamala Jayanthi, P.D., Kempraj, V., Aurade, R.M., Venkataramanappa, R.K., Nandagopal, B., Verghese, A., Bruce, T.J.A., 2014. Specific volatile compounds from mango elicit oviposition in gravid Bactrocera dorsalis females. J. Chem. Ecol. 40, 259-266. doi:10.1007/s10886-014-0403-7

Kamala Jayanthi, P.D., Woodcock, C.M., Caulfield, J., Birkett, M.A., Bruce, T.J.A., 2012. Isolation and identification of host cues from mango, Mangifera indica, that attract gravid female oriental fruit fly, Bactrocera dorsalis. J. Chem. Ecol. 38, 361-369. doi:10.1007/s10886-012-0093-y

Kline, D.L., 1994. Olfactory attractants for mosquito surveillance and control: 1Octen-3-ol. J. Am. Mosq. Control Assoc. 10, 280-287.

Kohl, J., Huoviala, P., Jefferis, G.S.X.E., 2015. Pheromone processing in Drosophila. Curr. Opin. Neurobiol. 34, 149-157. doi:10.1016/j.conb.2015.06.009

Kreher, S.A., Mathew, D., Kim, J., Carlson, J.R., 2008. Translation of sensory input into behavioral output via an olfactory system. Neuron 59, 110-124. doi:10.1016/j.neuron.2008.06.010

Kurtovic, A., Widmer, A., Dickson, B.J., 2007. A single class of olfactory neurons mediates behavioural responses to a Drosophila sex pheromone. Nature 446, 542-546. doi:10.1038/nature 05672

Kwon, J.Y., Dahanukar, A., Weiss, L.A., Carlson, J.R., 2007. The molecular basis of CO2 reception in Drosophila. Proc. Natl. Acad. Sci. U.S.A. 104, 3574-3578. doi:10.1073/pnas.0700079104

Liu, C., Liu, Y., Guo, M., Cao, D., Dong, S., Wang, G., 2014. Narrow tuning of an odorant receptor to plant volatiles in Spodoptera exigua (Hübner). Insect Mol. Biol. 23, 487-496. doi:10.1111/imb.12096

Liu, Z., Smagghe, G., Lei, Z., Wang, J.J., 2016. Identification of male- and female-specific olfaction genes in antennae of the oriental fruit fly (Bactrocera dorsalis). PLoS One 11(2): e0147783. doi:10.1371/journal.pone.0147783

Lu, T., Qiu, Y.T., Wang, G., Kwon, J.Y., Rutzler, M., Kwon, H.W., Pitts, R.J., van Loon, J.J.A., Takken, W., Carlson, J.R., Zwiebel, L.J., 2007. Odor coding in the maxillary palp of the malaria vector mosquito Anopheles gambiae. Curr. Biol. 17, 1533-1544. doi:10.1016/j.cub.2007.07.062 
825

826

827

828

829

830

831

832

833

834

835

836

837

838

839

840

841

842

843

844

845

846

847

848

849

850

851

852

853

854

855

856

857

Mansourian, S., Stensmyr, M.C., 2015. The chemical ecology of the fly. Curr. Opin. Neurobiol. 34, 95-102. doi:10.1016/j.conb.2015.02.006

Metcalf, R., 1990. Chemical ecology of Dacinae fruit flies (Diptera: Tephritidae). Ann. Entomol. Soc. Am. 83, 1017-1030.

Mitsuno, H., Sakurai, T., Murai, M., Yasuda, T., Kugimiya, S., Ozawa, R., Toyohara, H., Takabayashi, J., Miyoshi, H., Nishioka, T., 2008. Identification of receptors of main sex-pheromone components of three Lepidopteran species. Eur. J. Neurosci. 28, 893-902. doi:10.1111/j.14609568.2008.06429.x

Miura, N., Nakagawa, T., Tatsuki, S., Touhara, K., Ishikawa, Y., 2009. A malespecific odorant receptor conserved through the evolution of sex pheromones in Ostrinia moth species. Int. J. Biol. Sci. 5, 319-330. doi:10.7150/ijbs.5.319

Miura, N., Nakagawa, T., Touhara, K., Ishikawa, Y., 2010. Broadly and narrowly tuned odorant receptors are involved in female sex pheromone reception in Ostrinia moths. Insect Biochem. Mol. Biol. 40, 64-73. doi:10.1016/j.ibmb.2009.12.011

Nakagawa, T., Sakurai, T., Nishioka, T., Touhara, K., 2005. Insect sexpheromone signals mediated by specific combinations of olfactory receptors. Science 307, 1638-1642. doi:10.1126/science.1106267

Nishida, R., 2002. Sequestration of defensive substances from plants by Lepidoptera. Annu. Rev. Entomol. 47, 57-92. doi:10.1146/annurev.ento.47.091201.145121

Nishida, R., Tan, K.H., Serit, M., Lajis, N.H., Sukari, A.M., Takahashi, S., Fukami, H., 1988. Accumulation of phenylpropanoids in the rectal glands of males of the Oriental fruit fly, Dacus dorsalis. Experientia 44, 534-536.

Nishida, R., and Tan, K.H., 2016. Search for new fruit fly attractants from plants: A review. Proceedings of the 9th International Symposium on Fruit Flies of Economic Importance, 249-262. ISBN: 978-616-358-207-2.

Opitz, S.E.W., Müller, C., 2009. Plant chemistry and insect sequestration. Chemoecology 19, 117-154. doi:10.1007/s00049-009-0018-6

Ozaki, K., Ryuda, M., Yamada, A., Utoguchi, A., Ishimoto, H., Calas, D., Marion-Poll, F., Tanimura, T., Yoshikawa, H., 2011. A gustatory receptor 
858

859

860

861

862

863

864

865

866

867

868

869

870

871

872

873

874

875

876

877

878

879

880

881

882

883

884

885

886

887

888

889

890

involved in host plant recognition for oviposition of a swallowtail butterfly. Nat. Commun. 2, 542. doi:10.1038/ncomms1548

Reddy, G.V.P., Guerrero, A., 2004. Interactions of insect pheromones and plant semiochemicals. Trends Plant Sci. 9, 253-261.

doi:10.1016/j.tplants.2004.03.009

Ritz, C., Baty, F., Streibig, J.C., Gerhard, D., 2015. Dose-response analysis using R. PLoS One 10(12): e0146021, 1-13. doi:10.1371/journal.pone.0146021

Rytz, R., Croset, V., Benton, R., 2013. Ionotropic Receptors (IRs): Chemosensory ionotropic glutamate receptors in Drosophila and beyond. Insect Biochem. Mol. Biol. 43, 888-897. doi:10.1016/j.ibmb.2013.02.007

Sakurai, T., Nakagawa, T., Mitsuno, H., Mori, H., Endo, Y., Tanoue, S., Yasukochi, Y., Touhara, K., Nishioka, T., 2004. Identification and functional characterization of a sex pheromone receptor in the silkmoth Bombyx mori. Proc. Natl. Acad. Sci. U.S.A. 101, 16653-16658. doi:10.1073/pnas.0407596101

Sato, K., Pellegrino, M., Nakagawa, T., Nakagawa, T., Vosshall, L.B., Touhara, K., 2008. Insect olfactory receptors are heteromeric ligand-gated ion channels. Nature 452, 1002-1006. doi:10.1038/nature06850

Sato, K., Tanaka, K., Touhara, K., 2011. Sugar-regulated cation channel formed by an insect gustatory receptor. Proc. Natl. Acad. Sci. U.S.A. 108, 1168011685. doi:10.1073/pnas.1019622108

Scott, K., Brady, R., Cravchik, A., Morozov, P., Rzhetsky, A., Zuker, C., Axel, R., 2001. A chemosensory gene family encoding candidate gustatory and olfactory receptors in Drosophila. Cell 104, 661-673. doi:10.1016/S00928674(01)00263-X

Shelly, T., 2010. Effects of methyl eugenol and raspberry ketone/cue lure on the sexual behavior of Bactrocera species ( Diptera : Tephritidae ). Appl. Entomol. Zool. 45, 349-361. doi:10.1303/aez.2010.349

Siderhurst, M.S., Jang, E.B., 2006a. Attraction of female oriental fruit fly, Bactrocera dorsalis, to Terminalia catappa fruit extracts in wind tunnel and olfactometer tests. Formos. Entomol. 26, 45-55.

Siderhurst, M.S., Jang, E.B., 2006b. Female-biased attraction of oriental fruit fly, Bactrocera dorsalis (Hendel), to a blend of host fruit volatiles from 
891

892

893

894

895

896

897

898

899

900

901

902

903

904

905

906

907

908

909

910

911

912

913

914

915

916

917

918

919

920

921

922

923

924

Terminalia catappa L. J. Chem. Ecol. 32, 2513-2524. doi:10.1007/s10886006-9160-6

Tamura, K., Peterson, D., Peterson, N., Stecher, G., Nei, M., Kumar, S., 2011. MEGA5: Molecular evolutionary genetics analysis using maximum likelihood, evolutionary distance, and maximum parsimony methods research resource. Mol. Biol. Evol. 28, 2731-2739. doi:10.1093/molbev/msr121

Tan, K.H., Nishida, R., 1996. Sex pheromone and mating competition after methyl eugenol consumption in the Bactrocera dorsalis complex. In: McPheron, B.A., Steck, G.A., (eds) Fruit fly pests: a world assessment of their biology and management. St. Lucie Press, Delray Beach, FL, pp 147153.

Tan, K.H., Nishida, R., 2012. Methyl eugenol : Its occurrence , distribution , and role in nature, especially in relation to insect behavior and pollination. $J$. Insect Sci. 12, 1-60.

Thompson, J.D., Higgins, D.G., Gibson, T.J., 1994. CLUSTAL W: improving the sensitivity of progressive multiple sequence alignment through sequence weighting, position-specific gap penalties and weight matrix choice. Nucleic Acids Res. 22, 4673-4680.

Thorne, N., Amrein, H., 2008. Atypical expression of Drosophila gustatory receptor genes in sensory and central neurons. J. Comp. Neurol. 506, 548568. doi:10.1002/cne

Touhara, K., Vosshall, L.B., 2009. Sensing odorants and pheromones with chemosensory receptors. Annu. Rev. Physiol. 71, 307-332. doi:10.1146/annurev.physiol.010908.163209

Vosshall, L.B., Stocker, R.F., 2007. Molecular architecture of smell and taste in Drosophila. Annu. Rev. Neurosci. 30, 505-533. doi:10.1146/annurev.neuro.30.051606.094306

Vosshall, L.B., Wong, A.M., Axel, R., 2000. An olfactory sensory map in the fly brain. Cell 102, 147-159. doi:10.1016/S0092-8674(00)00021-0

Wallingford, A.K., Hesler, S.P., Cha, D.H., Loeb, G.M., 2016. Behavioral response of spotted-wing drosophila, Drosophila suzukii Matsumura, to aversive odors and a potential oviposition deterrent in the field. Pest Manag. Sci. 72, 701-706. doi:10.1002/ps.4040 
925

926

927

928

929

930

931

932

933

934

935

936

937

938

939

940

941

942

943

944

945

946

947

948

949

950

951

952

Wang, G., Carey, A.F., Carlson, J.R., Zwiebel, L.J., 2010. Molecular basis of odor coding in the malaria vector mosquito Anopheles gambiae. Proc. Natl. Acad. Sci. 107, 4418-4423. doi:10.1073/pnas.0913392107

Wanner, K.W., Nichols, A.S., Allen, J.E., Bunger, P.L., Garczynski, S.F., Linn, C.E., Robertson, H.M., Luetje, C.W., 2010. Sex pheromone receptor specificity in the European corn borer moth, Ostrinia nubilalis. PLoS One 5(1): e8685. doi:10.1371/journal.pone.0008685

Wicher, D., Schäfer, R., Bauernfeind, R., Stensmyr, M.C., Heller, R., Heinemann, S.H., Hansson, B.S., 2008. Drosophila odorant receptors are both ligand-gated and cyclic-nucleotide- activated cation channels. Nature 452, 1007-1011. doi:10.1038/nature06861

Wu, Z., Zhang, H., Wang, Z., Bin, S., He, H., Lin, J., 2015. Discovery of chemosensory genes in the oriental fruit fly, Bactrocera dorsalis. PLoS One 10(6): e0129794. doi:10.1371/journal.pone.0129794

Yang, B., Ozaki, K., Ishikawa, Y., Matsuo, T., 2015. Identification of candidate odorant receptors in asian corn borer Ostrinia furnacalis. PLoS One 10(3): e0121261. doi:10.1371/journal.pone.0121261

Zawistowski, S., Richmond, R.C., 1986. Inhibition of courtship and mating of Drosophila melanogaster by the male-produced lipid, cis-vaccenyl acetate. J. Insect Physiol. 32, 189-192. doi:10.1016/0022-1910(86)90057-0

Zhang, R., Wang, B., Grossi, G., Falabella, P., Liu, Y., Yan, S., Lu, J., Xi, J., Wang, G., 2017. Molecular basis of alarm pheromone detection in aphids. Curr. Biol. 27, 55-61. doi:10.1016/j.cub.2016.10.013

Zheng, W., Zhu, C., Peng, T., Zhang, H., 2012. Odorant receptor co-receptor Orco is upregulated by methyl eugenol in male Bactrocera dorsalis (Diptera: Tephritidae). J. Insect Physiol. 58, 1122-1127. doi:10.1016/j.jinsphys.2012.05.011 


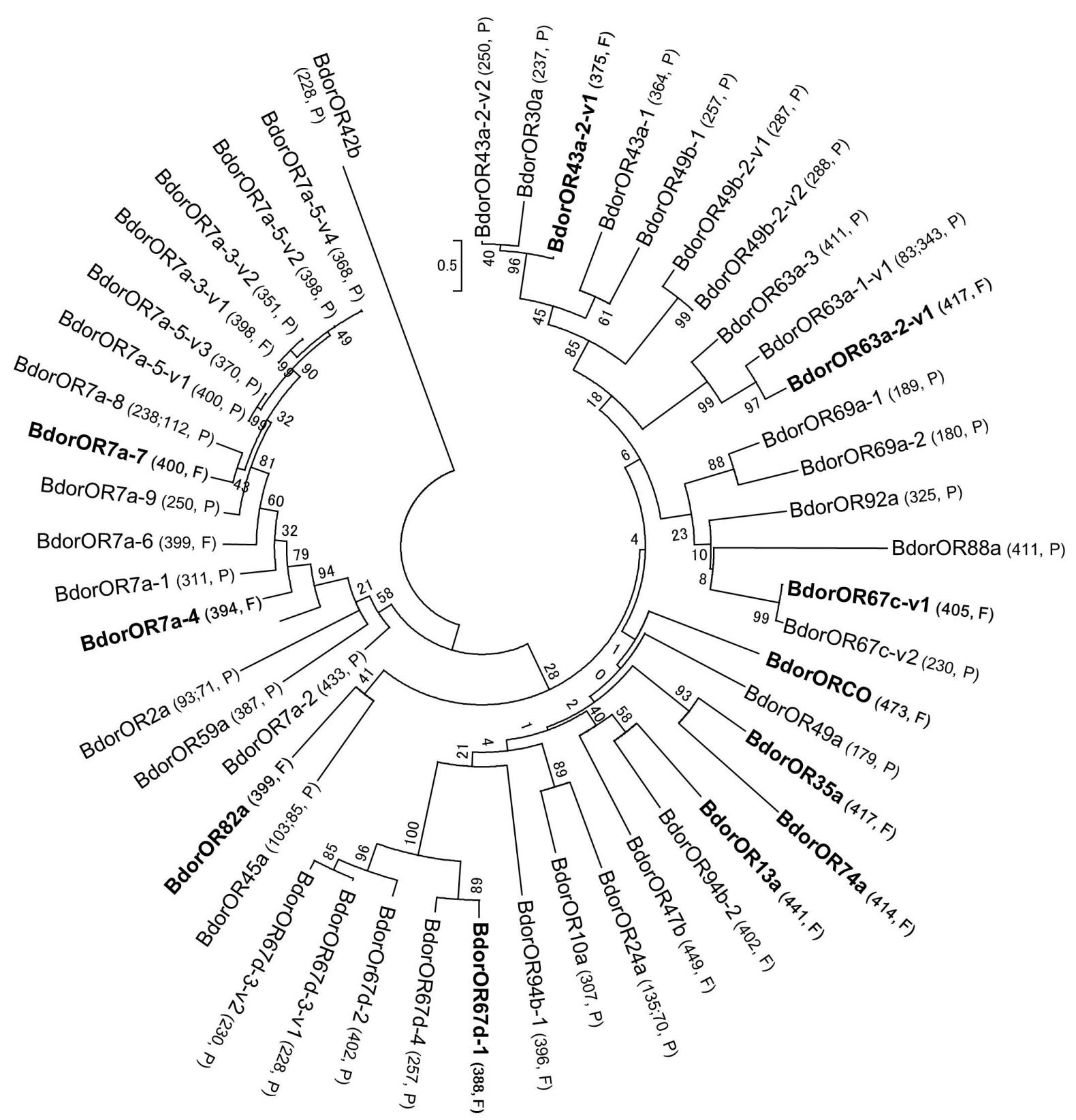



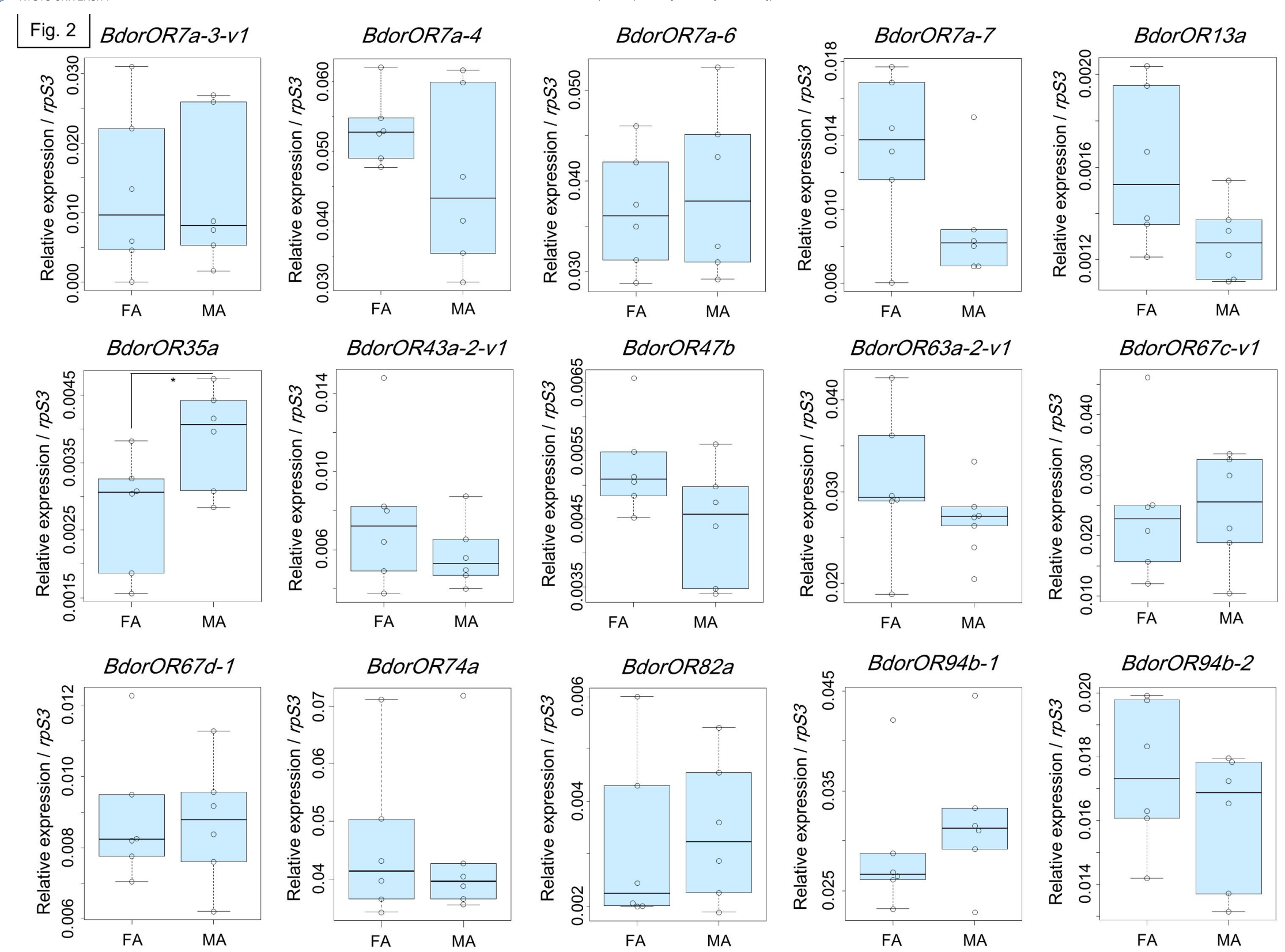


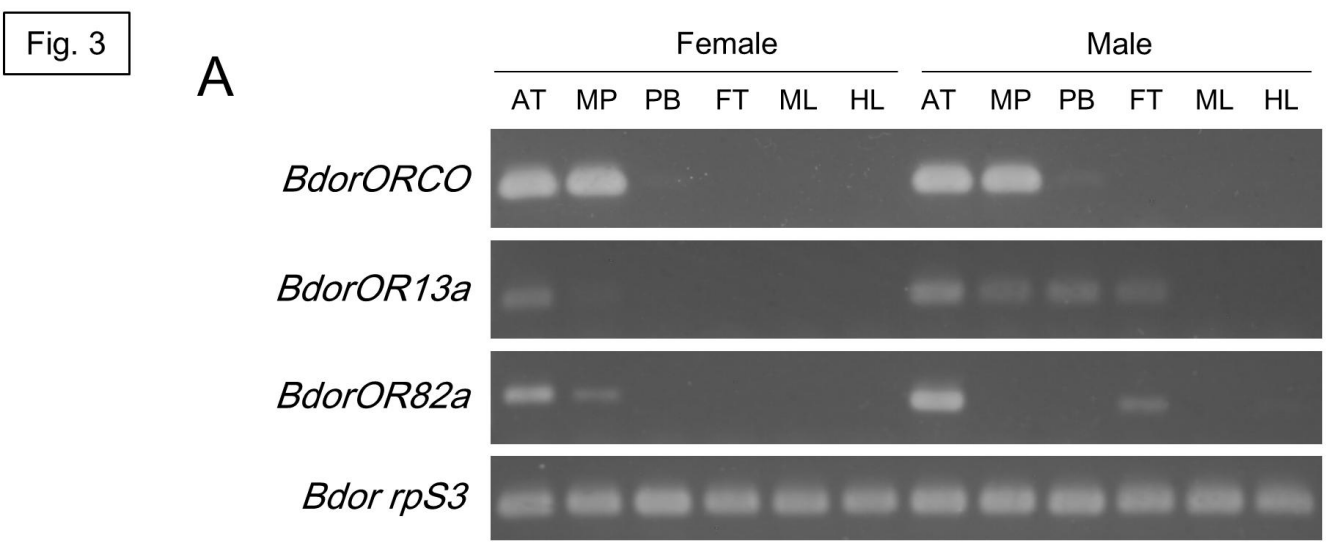

B

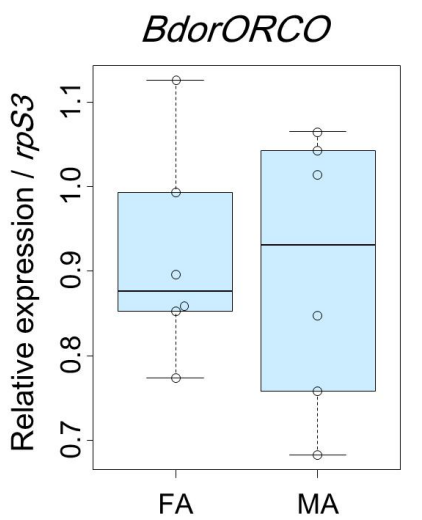

C

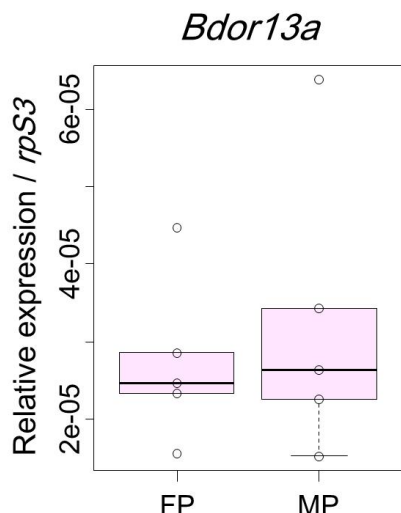

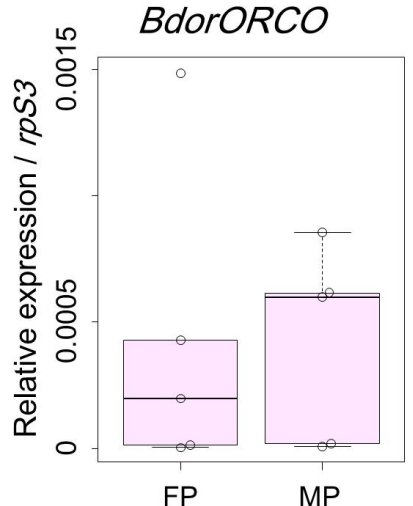

Bdor13a

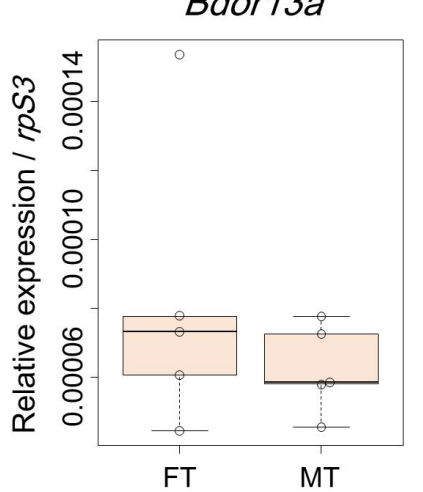

BdorORCO
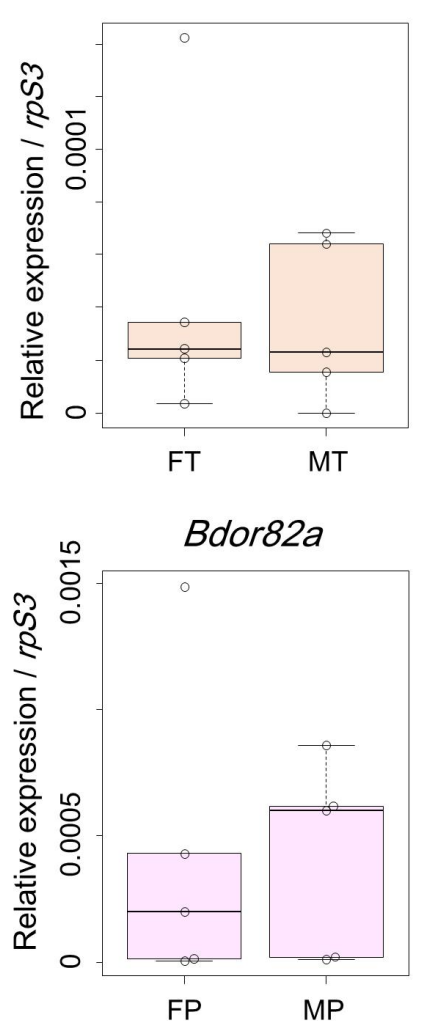

Bdor82a

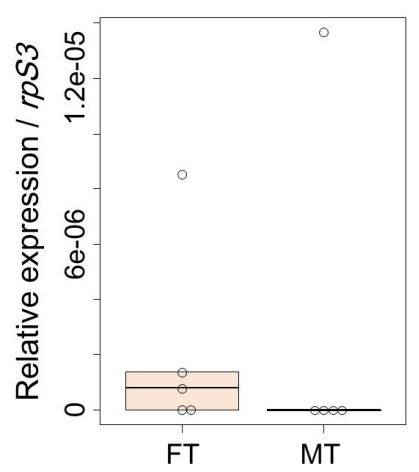




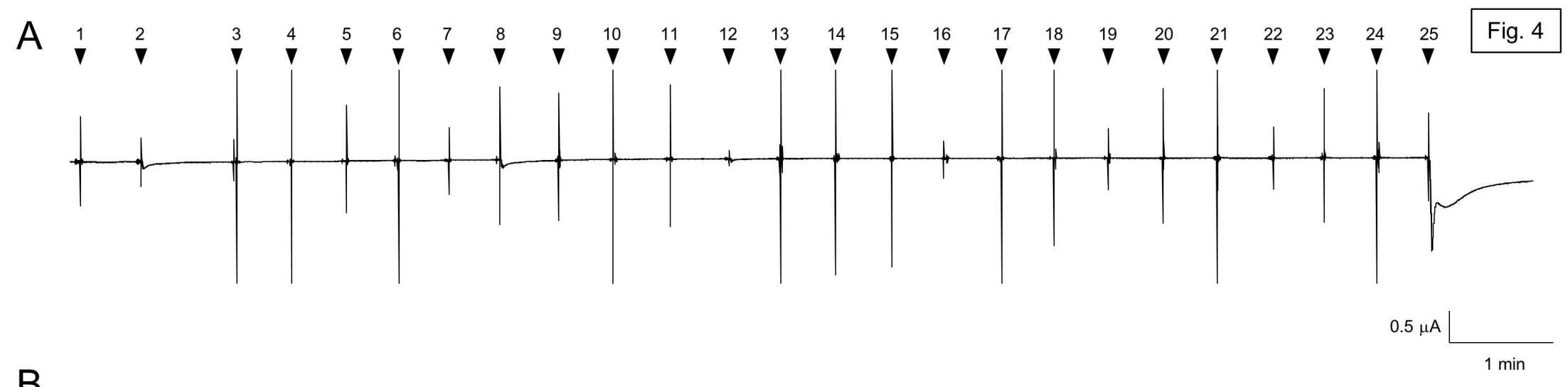

B

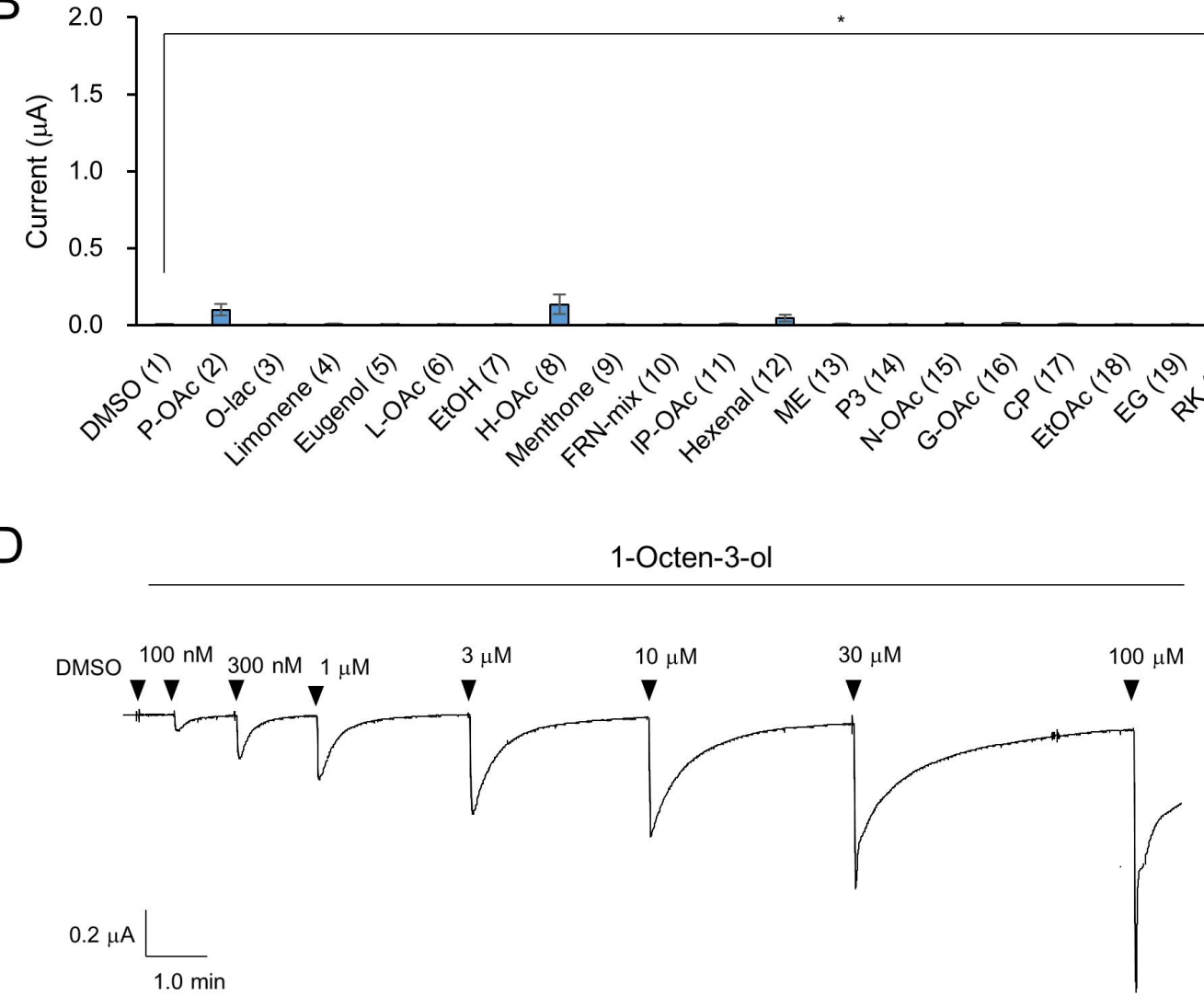

C<smiles>C=CC(O)CCCCC</smiles>

1-Octen-3-ol
E

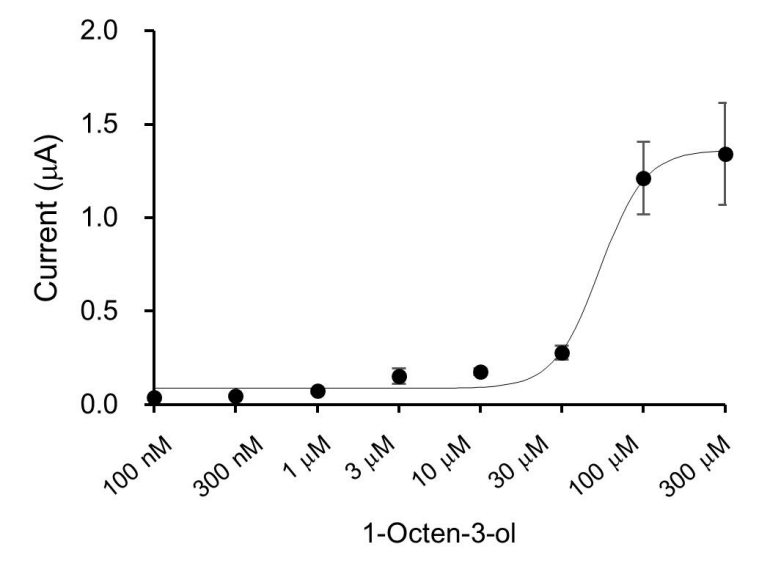



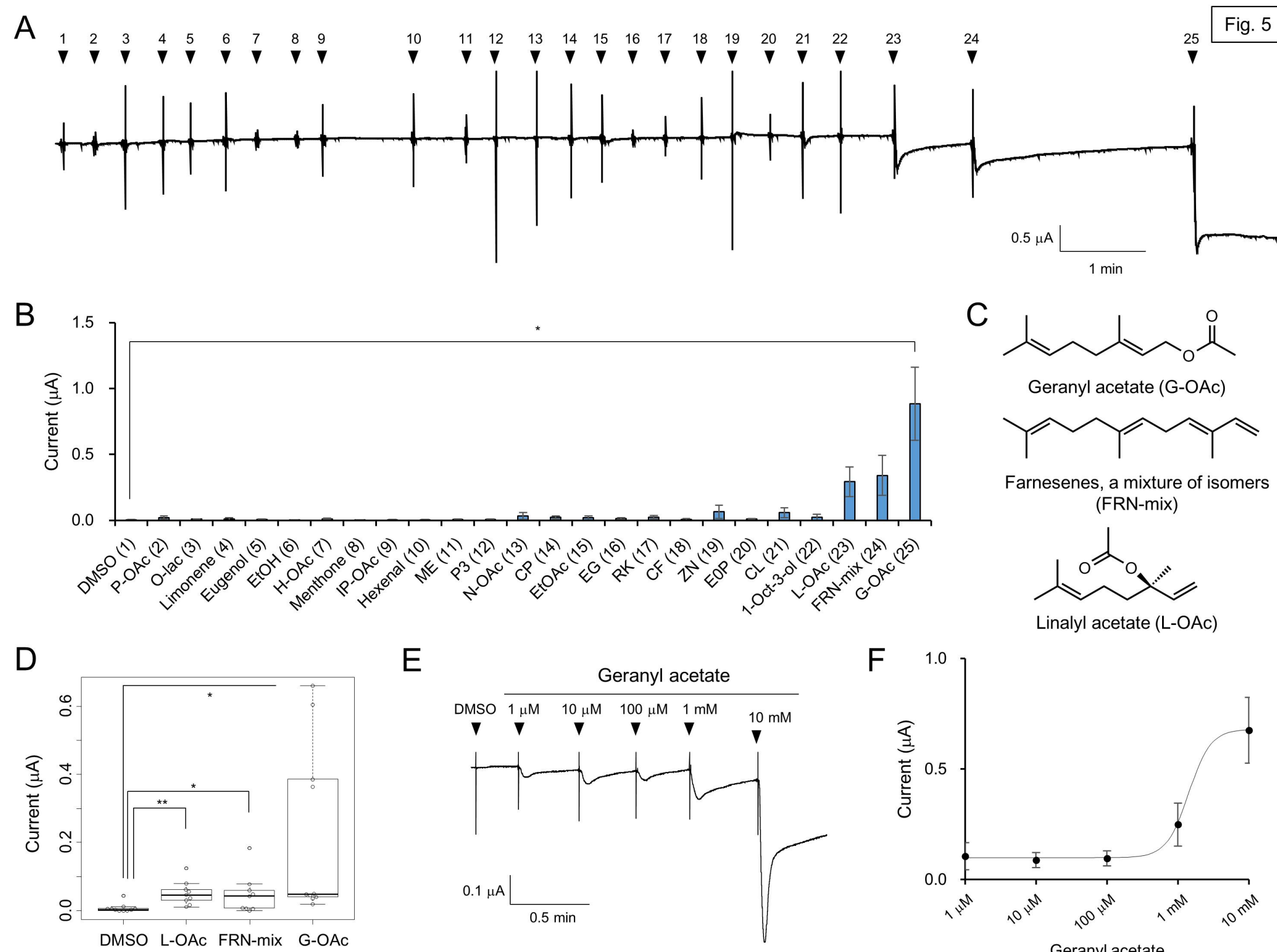

E
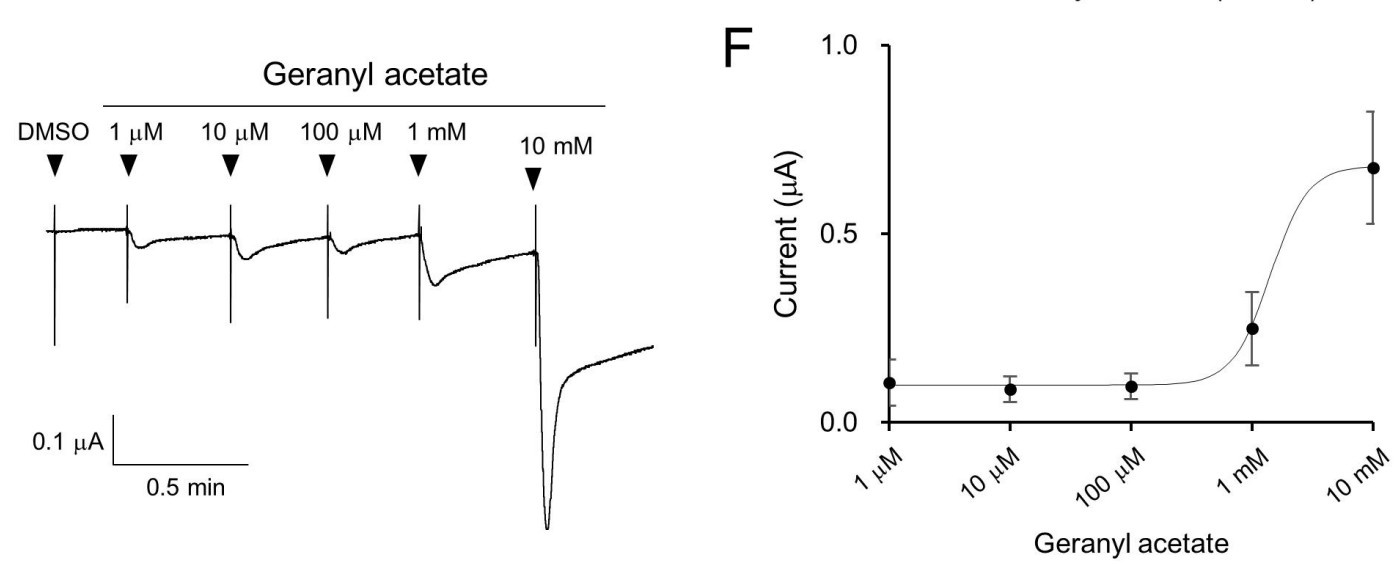


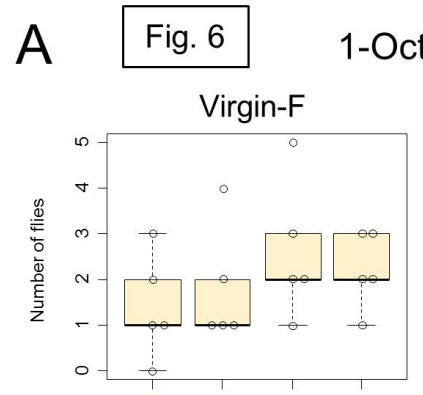

T-G) T-W $\quad C . G \quad C . W$

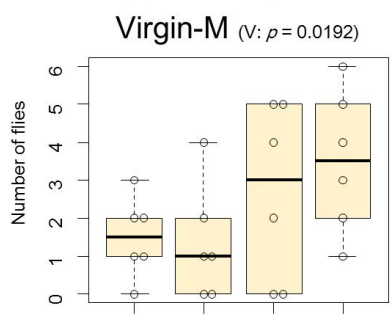

T.G) T-W $\quad$ C. $\quad$ C $\quad$ C.W

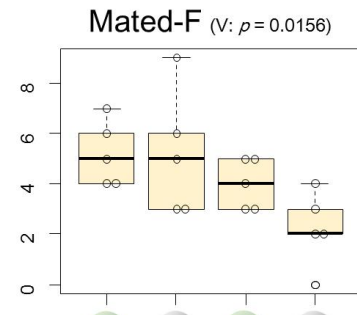

T-G) T-W C-G C.W

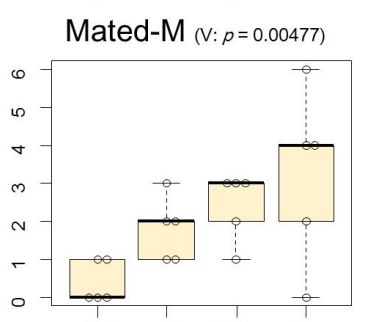

T-G) $\quad T-W \quad C-G \quad C-W$

Farnesenes

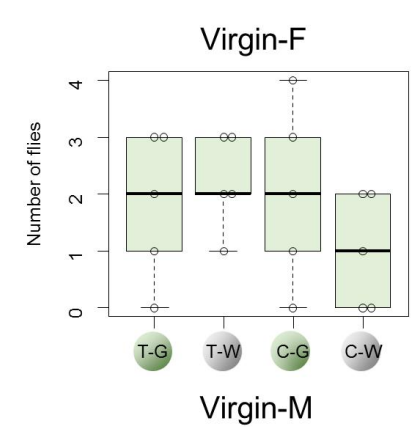

Mated-F $(v: p=0.0119)$

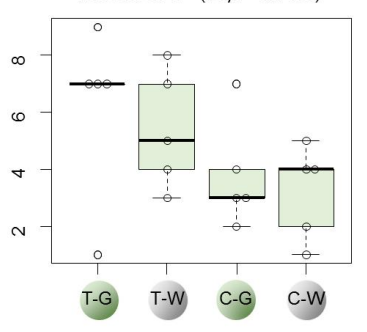

Mated-M (v: $p=0.0476)$
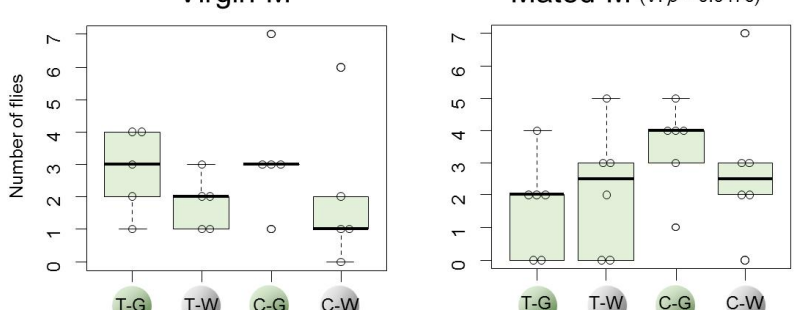

T-G) $\quad T-W \quad C-G \quad C-W$

Geranyl acetate
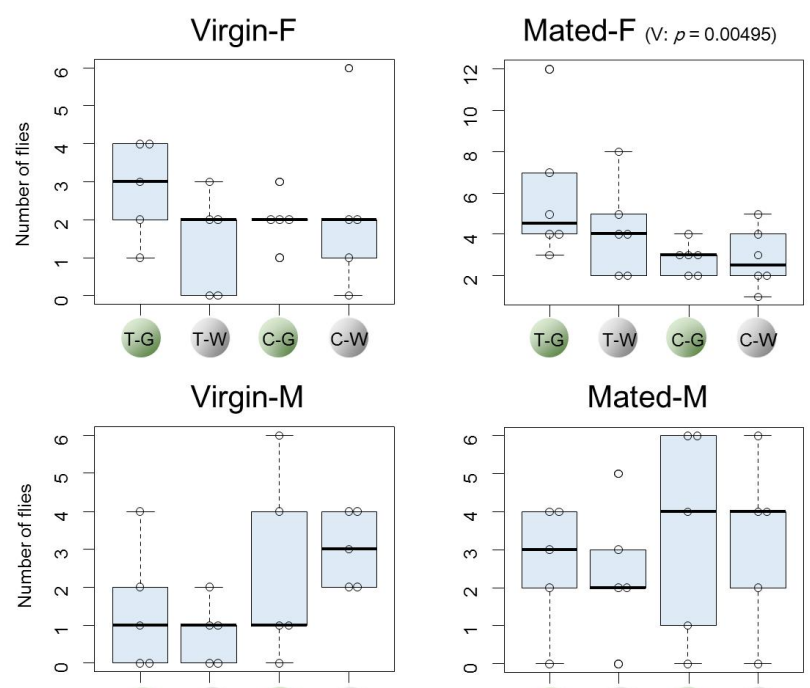

T.C) T-W $\quad C-6$ C.W

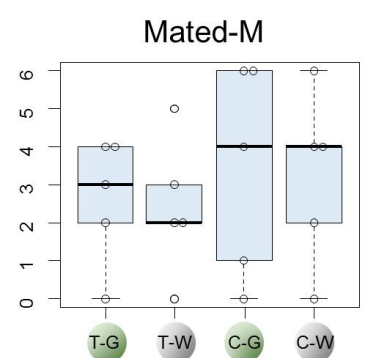

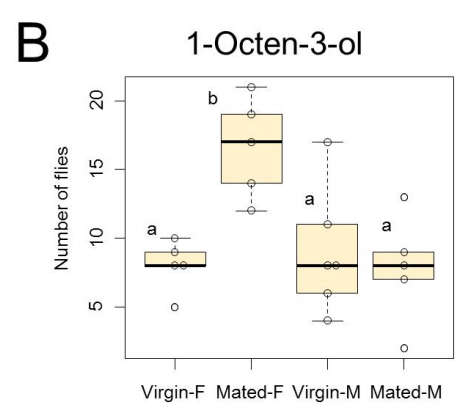

T-G T-W C-G C-W

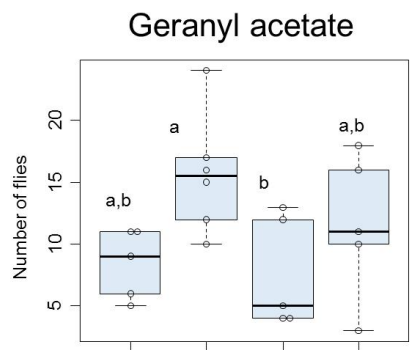

Virgin-F Mated-F Virgin-M Mated-M

Linalyl acetate

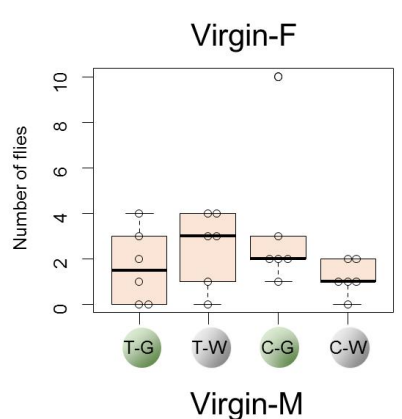

Mated-F (c: $p=0.0253)$
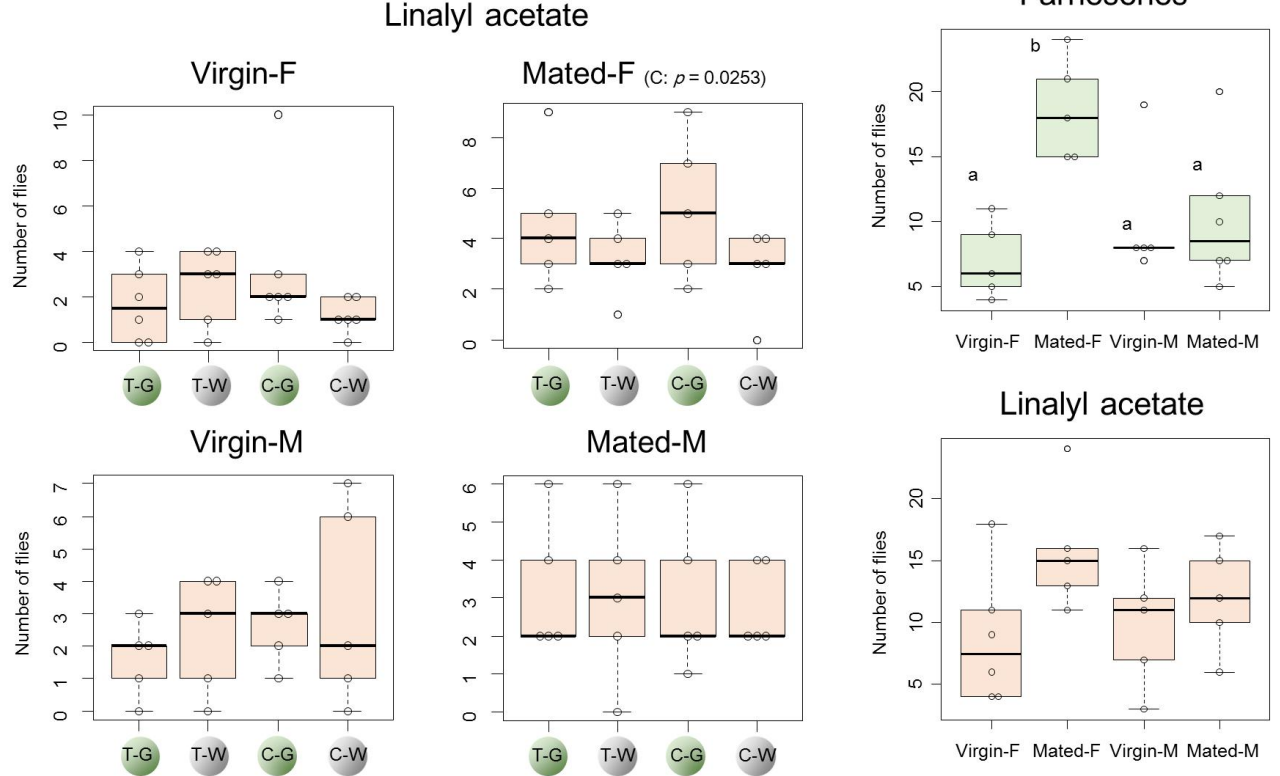

Linalyl acetate

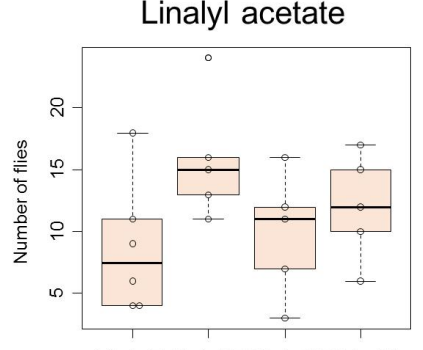

Virgin-F Mated-F Virgin-M Mated-M 
Table 1. Summary of sequence data analysis.

\begin{tabular}{lcc}
\hline & Antenna & Proboscis \\
\hline Number of raw reads & $1,675,116$ & $2,159,685$ \\
Number of clean reads & $1,159,879$ & $1,383,389$ \\
Number of assembled contigs & \multicolumn{2}{c}{71,766} \\
Mean length of contigs (bp) & \multicolumn{2}{c}{486} \\
\hline
\end{tabular}


Table 2. Candidate chemosensory receptor genes identified from the transcriptome.

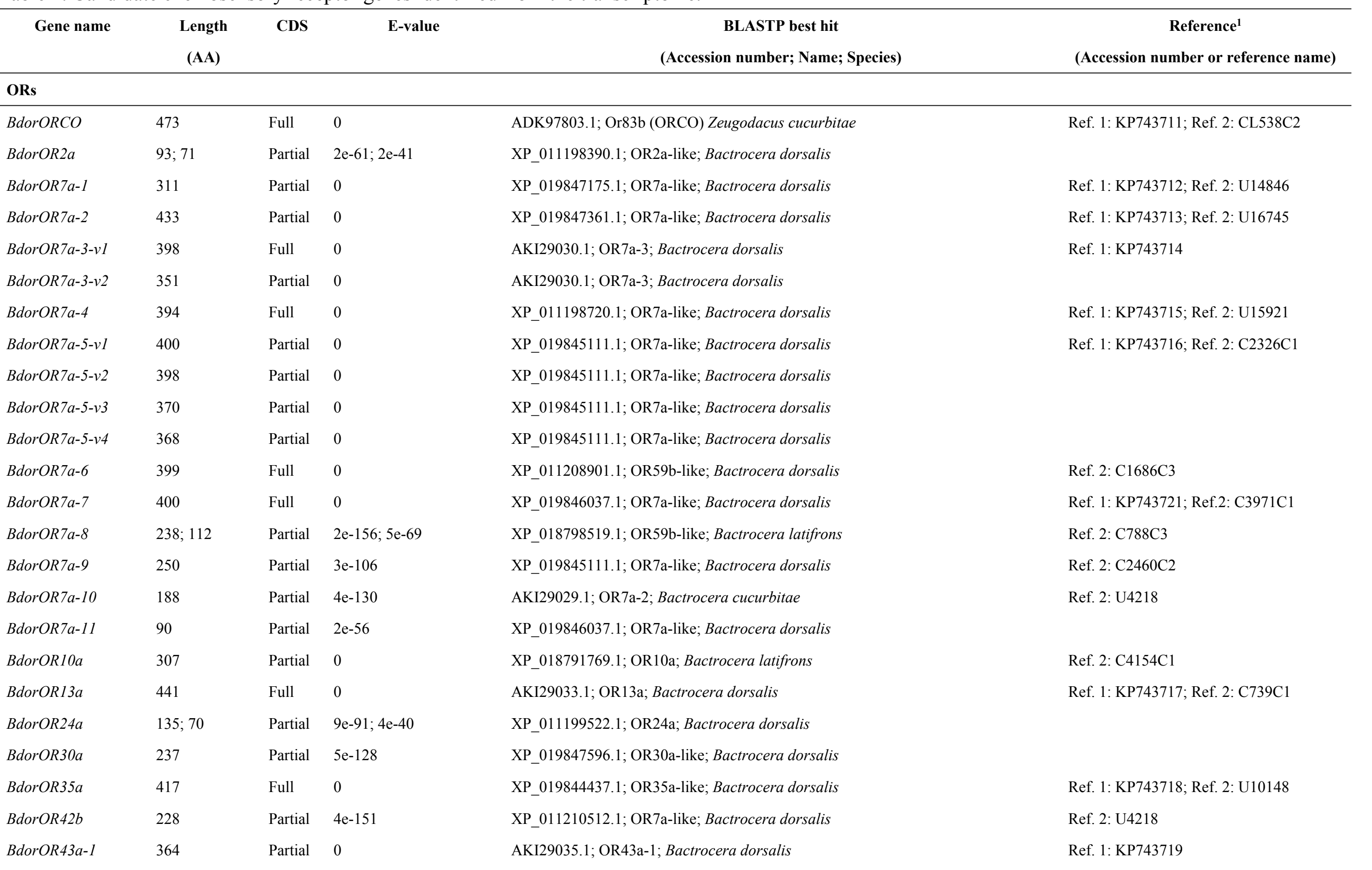




\begin{tabular}{|c|c|c|c|}
\hline BdorOR43a-2-v1 & 375 & Full & 0 \\
\hline BdorOR43a-2-v2 & 250 & Partial & $4 e-179$ \\
\hline BdorOR43a-3 & 72 & Partial & $1 e-37$ \\
\hline BdorOR45a & $103 ; 85$ & Partial & $8 e-66 ; 6 e-54$ \\
\hline BdorOR47b & 449 & Full & 0 \\
\hline BdorOR49a & 179 & Partial & $1 \mathrm{e}-126$ \\
\hline BdorOR49b-1 & 257 & Partial & 0 \\
\hline BdorOR49b-2-v1 & 287 & Partial & $2 \mathrm{e}-170$ \\
\hline BdorOR49b-2-v2 & 288 & Partial & 0 \\
\hline BdorOR $49 b-3$ & 105 & Partial & $2 \mathrm{e}-67$ \\
\hline BdorOR49b-4 & 83 & Partial & $4 e-50$ \\
\hline BdorOR59a & 387 & Partial & 0 \\
\hline BdorOR63a-1-v1 & $83 ; 343$ & Partial & $1 \mathrm{e}-49 ; 0$ \\
\hline BdorOR63a-1-v2 & 52 & Partial & $2 \mathrm{e}-21$ \\
\hline BdorOR63a-1-v3 & 82 & Partial & $6 e-48$ \\
\hline BdorOR63a-2-v1 & 417 & Full & 0 \\
\hline BdorOR63a-2-v2 & 139 & Partial & $4 e-70$ \\
\hline BdorOR63a-3 & 411 & Partial & 0 \\
\hline BdorOR67c-v1 & 405 & Full & 0 \\
\hline BdorOR67c-v2 & 230 & Partial & $2 \mathrm{E}-152$ \\
\hline BdorOR67d-1 & 388 & Full & 0 \\
\hline BdorOR67d-2 & 402 & Partial & $4 e-178$ \\
\hline BdorOR67d-3-v1 & 228 & Partial & $2 \mathrm{e}-133$ \\
\hline BdorOR67d-3-v2 & 230 & Partial & $6 e-138$ \\
\hline BdorOR67d-4 & 257 & Partial & 0 \\
\hline BdorOR69a-1 & 189 & Partial & $3 e-133$ \\
\hline BdorOR69a-2 & 180 & Partial & $3 e-103$ \\
\hline BdorOR69a-3 & 69 & Partial & $5 e-42$ \\
\hline BdorOR74a & 414 & Full & 0 \\
\hline
\end{tabular}

AKI29036.1; OR43a-2; Bactrocera dorsalis

Ref. 1: KP743720

XP_019847608.1; Or2-like; Bactrocera dorsalis

Ref. 2: C3544C

XP 014097484.1; Or2-like; Bactrocera oleae

XP_011212447.2; OR45a-like; Bactrocera dorsalis

XP 019847427.1; OR47b; Bactrocera dorsalis

XP 011212431.1; OR49a-like; Bactrocera dorsalis

Ref. 2: U11993

XP_019845516.1; OR49b; Bactrocera dorsalis

Ref. 1: KP743723; Ref. 2: C6087C2

XP_019847679.1; OR49b-like; Bactrocera dorsalis

XP_019847679.1; OR49b-like; Bactrocera dorsalis

Ref. 1: KP743724

AKI29039.1; OR49b-1; Bactrocera dorsalis

XP 019847607.1; OR2-like; Bactrocera dorsalis

AKI29041.1; OR59a; Bactrocera dorsalis

Ref. 1: KP743725; Ref. 2: U350

AKI29042.1; OR63a-1; Bactrocera dorsalis

AKI29042.1; OR63a-1; Bactrocera dorsalis

Ref. 1: KP743726

XP 018787905.1; OR63a-like; Bactrocera latifrons

AKI29043.1; OR63a-2; Bactrocera dorsalis

Ref. $1 \cdot$ KP743727; Ref. $2 \cdot$ U11167

XP 019847162.1; OR63a-like; Bactrocera dorsalis XP_018783180.1; OR63a; Bactrocera latifrons XP_011200400.1; OR67c-like; Bactrocera dorsalis XP 011200401.1; OR67c-like; Bactrocera dorsalis XP_011203703.1; OR67d-like; Bactrocera dorsalis XP_017473047.1; OR67d-like; Rhagoletis zephyria XP_017473047.1; OR67d-like; Rhagoletis zephyria XP_017473047.1; OR67d-like; Rhagoletis zephyria XP 011203704.2; OR67d-like; Bactrocera dorsalis AKI29046.1; OR69a; Bactrocera dorsalis

XP 011191113.1; OR69a isoformA; Bactrocera cucurbitae

Ref. 2: U1859

Ref. 1: KP743728; Ref. 2: C173C1

Ref. 1: KP743729; Ref. 2: C8295C

Ref. 2: U33

Ref. 2: U3061

Ref. 1: KP743730

Ref. 2: U12022

XP_011209369.1; putative OR69a; Bactrocera dorsalis

XP_011201924.2; OR74a-like; Bactrocera dorsalis

Ref. 1: KP743731 


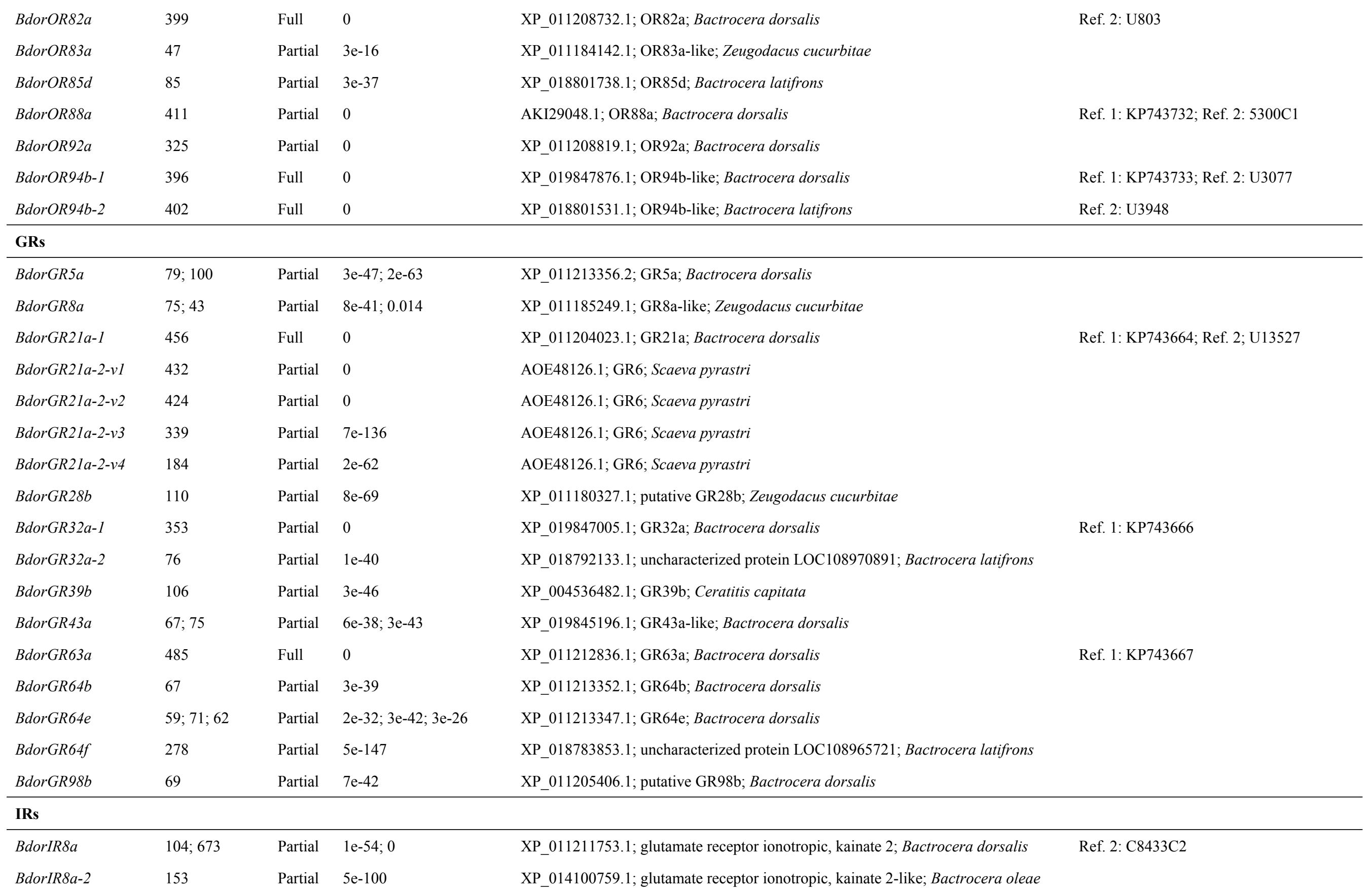




\begin{tabular}{|c|c|c|c|}
\hline BdorIR25a & 940 & Full & 0 \\
\hline BdorIR31a-1 & 108 & Partial & $1 \mathrm{e}-54$ \\
\hline BdorIR31a-2 & 83 & Partial & $1 e-27$ \\
\hline BdorIR40a & $128 ; 265 ; 83$ & Partial & $6 e-85 ; 0 ; 1 e-47$ \\
\hline BdorIR4la & $135 ; 87 ; 213$ & Partial & $5 e-91 ; 1 \mathrm{e}-48 ; 8 \mathrm{e}-147$ \\
\hline BdorIR56c & $118 ; 216$ & Partial & $1 \mathrm{e}-12 ; 4 \mathrm{e}-132$ \\
\hline BdorIR64a-1 & 322 & Partial & 0 \\
\hline BdorIR64a-2 & 96 & Partial & $1 \mathrm{e}-58$ \\
\hline BdorIR75a-1 & 342 & Partial & 0 \\
\hline BdorIR75a-2 & $140 ; 232$ & Partial & $2 \mathrm{e}-79 ; 2 \mathrm{e}-164$ \\
\hline BdorIR75b & 95 & Partial & $2 e-49$ \\
\hline BdorIR75d & $162 ; 105$ & Partial & $5 e-110 ; 3 e-64 ; 0$ \\
\hline BdorIR76a-1 & $137 ; 147$ & Partial & $7 e-92 ; 3 e-100$ \\
\hline BdorIR76a-2 & 286 & Partial & 0 \\
\hline BdorIR76b & 659 & Full & 0 \\
\hline BdorIR84a & 703 & Partial & 0 \\
\hline BdorIR92a-1 & $140 ; 116 ; 246$ & Partial & $3 e-85 ; 4 e-53 ; 4 e-177$ \\
\hline BdorIR92a-2 & 146 & Partial & 2e-92 \\
\hline BdorIR93a-1 & $93 ; 602$ & Partial & $7 e-55 ; 0$ \\
\hline BdorIR93a-2 & 76 & Partial & $1 e-38$ \\
\hline BdorIR94f & 95 & Partial & $3 e-58$ \\
\hline
\end{tabular}

XP_011207795.1; IR25a; Bactrocera dorsalis

XP_018804290.1, uncharacterized protein LOC108978446, Bactrocera latifrons

XP_012162538.1; LOC101456253, Ceratitis capitata

XP_011212457.2; uncharacterized protein LOC105232474; Bactrocera dorsalis AKI28986.1; IR41a; Bactrocera dorsalis

XP_018794909.1; uncharacterized protein LOC108972669; Bactrocera latifrons XP_018799073.1; uncharacterized protein LOC108975188; Bactrocera latifrons XP_019845172.1; uncharacterized protein LOC105224490; Bactrocera dorsalis XP_019845038.1; uncharacterized protein LOC109579404; Bactrocera dorsalis XP_019845037.1; glutamate receptor; Bactrocera dorsalis

XP_014088428.1; uncharacterized protein LOC106616338; Bactrocera oleae XP 019844868.1; uncharacterized protein LOC105223467; Bactrocera dorsalis XP_011204763.1; uncharacterized protein LOC105227219; Bactrocera dorsalis XP 014086277.1; uncharacterized protein LOC106614874; Bactrocera oleae AKI28988.1; IR76b; Bactrocera dorsalis

XP_011193628.1; glutamate receptor 1; Zeugodacus cucurbitae AKI28990.1; IR92a; Bactrocera dorsalis

XP_019845172.1; uncharacterized protein LOC105224490; Bactrocera dorsalis XP_011214752.1; glutamate receptor ionotropic, delta-1; Bactrocera dorsalis XP_014095980.1; uncharacterized protein LOC106621575; Bactrocera oleae XP_011199185.1; uncharacterized protein LOC105223232; Bactrocera dorsalis
Ref. 1: U215

Ref. 1: KP743669; Ref. 2: U9427

Ref. 1: KP743670

Ref. 2: U7132

Ref. 2: U14774

Ref. 1: KP743671

Ref. 1: KP743672; Ref. 2: C1154C3

Ref. 1: KP743673

Ref. 1: KP743674; Ref. 2: C2923C2

Ref. 2: U7132

${ }^{1}$ Homologs representing more than 90\% amino acid identities with chemosensory receptors identified in the present study are listed as references. Ref. 1: Wu et al., 2015 ; Ref.2: Liu et al., 2016. 
Table 3. Results of data analysis using a generalized linear model with a binomial logit fit to identify the factors that influenced the numbers of fruit flies landing on the spheres.

\begin{tabular}{|c|c|c|c|c|c|}
\hline Volatile & Fruit fly & Factor & AIC & Deviance & $P$ \\
\hline \multirow[t]{16}{*}{ 1-Octen-3-ol } & \multirow[t]{4}{*}{ Virgin female } & Null & 64.9 & - & - \\
\hline & & Volatile (V) & 65.2 & 1.68 & 0.195 \\
\hline & & Color (C) & 66.9 & 0.00 & 1.000 \\
\hline & & $\mathrm{V} \times \mathrm{C}$ & 67.2 & 1.68 & 0.432 \\
\hline & \multirow[t]{4}{*}{ Mated female } & Null & 85.0 & - & - \\
\hline & & Volatile (V) & 81.2 & 5.85 & $0.016^{*}$ \\
\hline & & Color (C) & 86.0 & 1.07 & 0.302 \\
\hline & & $\mathrm{V} \times \mathrm{C}$ & 82.1 & 6.92 & $0.031^{*}$ \\
\hline & \multirow[t]{4}{*}{ Virgin male } & Null & 79.3 & - & - \\
\hline & & Volatile (V) & 75.8 & 5.48 & $0.019^{*}$ \\
\hline & & Color (C) & 81.3 & 0.00 & 1.000 \\
\hline & & $\mathrm{V} \times \mathrm{C}$ & 77.8 & 5.48 & 0.065 \\
\hline & \multirow[t]{4}{*}{ Mated male } & Null & 73.9 & - & - \\
\hline & & Volatile (V) & 67.9 & 7.97 & $0.005 * *$ \\
\hline & & Color (C) & 72.6 & 3.27 & 0.071 \\
\hline & & $\mathrm{V} \times \mathrm{C}$ & 66.6 & 11.26 & $0.004 * *$ \\
\hline \multirow[t]{16}{*}{ Geranyl acetate } & \multirow[t]{4}{*}{ Virgin female } & Null & 71.4 & - & - \\
\hline & & Volatile (V) & 73.4 & 0.00 & 1.000 \\
\hline & & Color (C) & 72.5 & 0.90 & 0.343 \\
\hline & & $\mathrm{V} \times \mathrm{C}$ & 74.5 & 0.90 & 0.638 \\
\hline & \multirow[t]{4}{*}{ Mated female } & Null & 105.6 & - & - \\
\hline & & Volatile (V) & 99.7 & 7.90 & $0.005 * *$ \\
\hline & & Color (C) & 106.4 & 1.16 & 0.282 \\
\hline & & $\mathrm{V} \times \mathrm{C}$ & 100.5 & 9.06 & $0.011^{*}$ \\
\hline & \multirow[t]{4}{*}{ Virgin male } & Null & 77.4 & - & - \\
\hline & & Volatile (V) & 72.1 & 7.22 & $0.007 * *$ \\
\hline & & Color (C) & 79.4 & 0.00 & 1.000 \\
\hline & & $\mathrm{V} \times \mathrm{C}$ & 74.1 & 7.22 & $0.027^{*}$ \\
\hline & \multirow[t]{4}{*}{ Mated male } & Null & 88.5 & - & - \\
\hline & & Volatile (V) & 89.4 & 1.17 & 0.278 \\
\hline & & Color (C) & 90.5 & 0.07 & 0.787 \\
\hline & & $\mathrm{V} \times \mathrm{C}$ & 91.3 & 1.25 & 0.536 \\
\hline Farnesenes & Virgin female & Null & 65.3 & - & - \\
\hline
\end{tabular}




\begin{tabular}{|c|c|c|c|c|c|}
\hline & & Volatile (V) & 66.5 & 0.74 & 0.389 \\
\hline & & Color (C) & 67.0 & 0.27 & 0.606 \\
\hline & & $\mathrm{V} \times \mathrm{C}$ & 68.3 & 1.01 & 0.604 \\
\hline & Mated female & Null & 92.7 & - & - \\
\hline & & Volatile (V) & 88.4 & 6.33 & $0.012 *$ \\
\hline & & Color (C) & 94.2 & 0.58 & 0.446 \\
\hline & & $\mathrm{V} \times \mathrm{C}$ & 89.8 & 6.92 & $0.031 *$ \\
\hline & Virgin male & Null & 77.0 & - & - \\
\hline & & Volatile (V) & 78.6 & 0.34 & 0.562 \\
\hline & & Color (C) & 75.9 & 3.06 & 0.080 \\
\hline & & $\mathrm{V} \times \mathrm{C}$ & 77.6 & 3.40 & 0.183 \\
\hline & Mated male & Null & 98.4 & - & - \\
\hline & & Volatile (V) & 96.5 & 3.92 & $0.048^{*}$ \\
\hline & & Color (C) & 100.4 & 0.02 & 0.895 \\
\hline & & $\mathrm{V} \times \mathrm{C}$ & 98.5 & 3.94 & 0.139 \\
\hline Linalyl acetate & Virgin female & Null & 98.6 & - & - \\
\hline & & Volatile (V) & 100.5 & 0.08 & 0.777 \\
\hline & & Color (C) & 99.3 & 1.29 & 0.256 \\
\hline & & $\mathrm{V} \times \mathrm{C}$ & 101.3 & 1.37 & 0.504 \\
\hline & Mated female & Null & 90.2 & - & - \\
\hline & & Volatile (V) & 92.2 & 0.01 & 0.907 \\
\hline & & Color (C) & 87.2 & 5.01 & $0.025^{*}$ \\
\hline & & $\mathrm{V} \times \mathrm{C}$ & 89.2 & 5.02 & 0.081 \\
\hline & Virgin male & Null & 81.8 & - & - \\
\hline & & Volatile (V) & 82.0 & 1.75 & 0.186 \\
\hline & & Color (C) & 82.7 & 1.06 & 0.304 \\
\hline & & $\mathrm{V} \times \mathrm{C}$ & 83.0 & 2.80 & 0.246 \\
\hline & Mated male & Null & 77.4 & - & - \\
\hline & & Volatile (V) & 79.3 & 0.07 & 0.790 \\
\hline & & Color (C) & 79.3 & 0.07 & 0.790 \\
\hline & & $\mathrm{V} \times \mathrm{C}$ & 81.3 & 0.14 & 0.932 \\
\hline
\end{tabular}

${ }^{*} p<0.05,{ }^{* *} p<0.01$. 


\section{Supplemental figure captions}

2

3 Fig. S1. Chemical structures of tested compounds for the functional analysis of

4 candidate olfactory receptors (ORs).

5

Fig. S4. Effects of ethanol and visual cues on the landing behavior of mated

21 Bactrocera dorsalis females. The box plot shows 25-75\% (box), median (band

22 inside), and minima to maxima (whiskers). (A) Numbers of mated females landing on

23 the green or white spheres. The numbers of flies are plotted as dots $(n=7) . \mathrm{T}-\mathrm{G}, \mathrm{T}-\mathrm{W}$,

24 C-G, and C-W indicate volatile-treated green balls, volatile-treated white balls, 
26 table shows the results of data analysis using a generalized linear model with a

27 binomial logit fit to identify the factors that influenced the number of fruit flies

28 landing on the spheres. Neither the volatile nor the color of the spheres had a

29 significant effect ( $p$-values $>0.05)$. (B) Comparison of the total numbers of mated

30 females landing on the spheres calculated from Fig. 6A and S4A. We detected no

31 significant differences between the treatments at $p<0.05$ according to Tukey's HSD

32 test.

33 


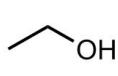

Ethanol (EtOH)

( $)$-1-Octen-3-ol (1-Oct-3-ol)
$\overbrace{0}^{H}$

(E)-2-Hexenal (Hexenal)

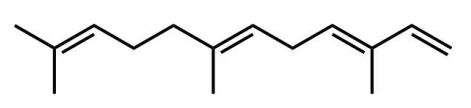

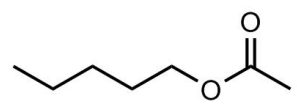

Pentyl acetate (P-OAc)

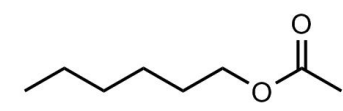

Hexyl acetate $(\mathrm{H}-\mathrm{OAc})$

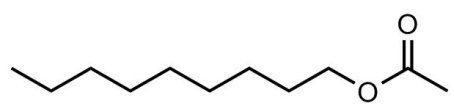

Nonyl acetate (N-OAc)

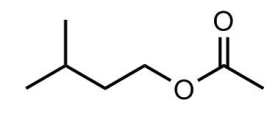

Isopentyl acetate (IP-OAc)

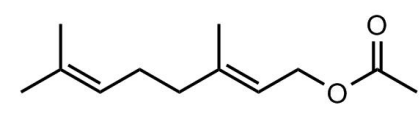

Geranyl acetate (G-OAc)

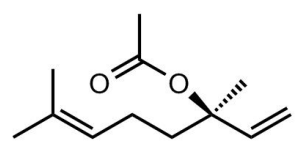

Linalyl acetate (L-OAc)

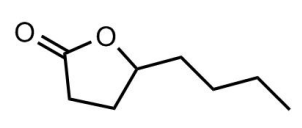

( \pm )- $\gamma$-Octalactone (O-lac)

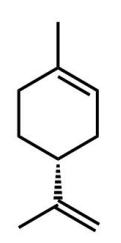

(R)-(+)-Limonene (Limonene)

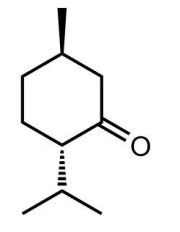

(-)-Menthone (Menthone)

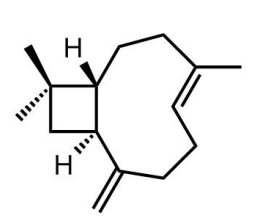

$\beta$-Caryophyllene (CP)

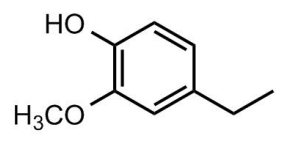

4-Ethylguaiacol (EG)

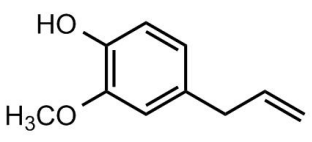

Eugenol

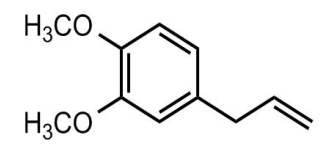

Methyl eugenol (ME)

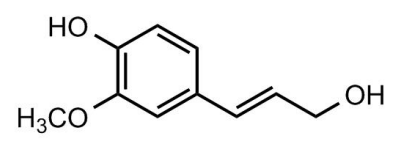

(E)-Coniferyl alcohol (CF)

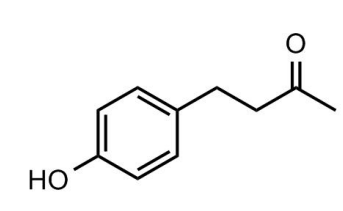

Raspberry ketone (RK)

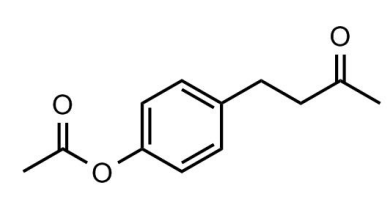

Cue-lure (CL)<smiles>COc1cc(CCC(C)=O)ccc1O</smiles>

Zingerone (ZN)

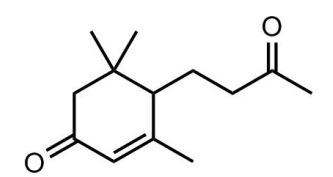

( \pm )-3-Oxo-7,8-dihydro- $\alpha$-ionone ( \pm )-4-Propionyloxyisophorone

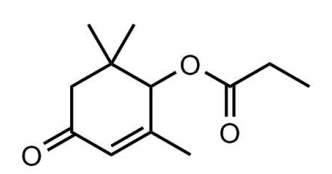

(EOP) 


\section{Fig. S2}

\section{A}
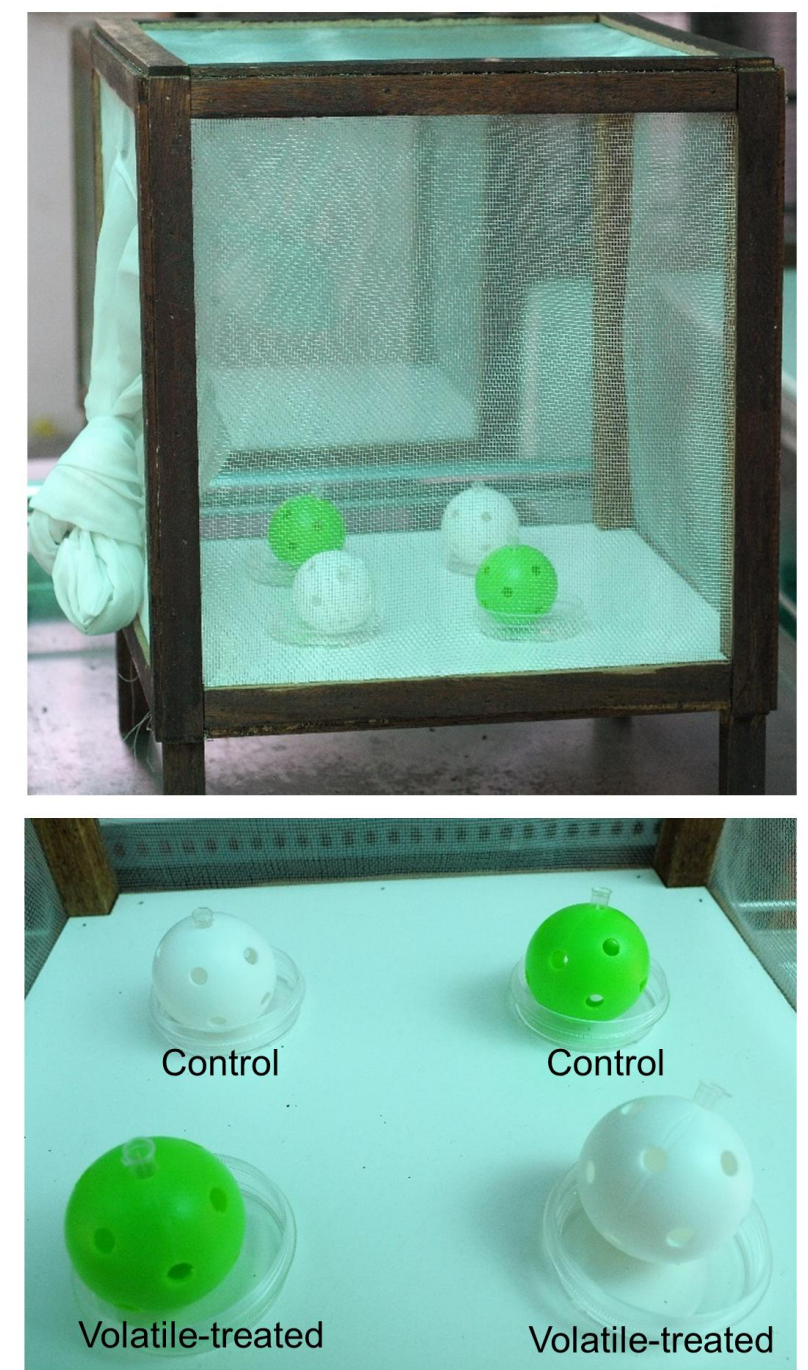

B
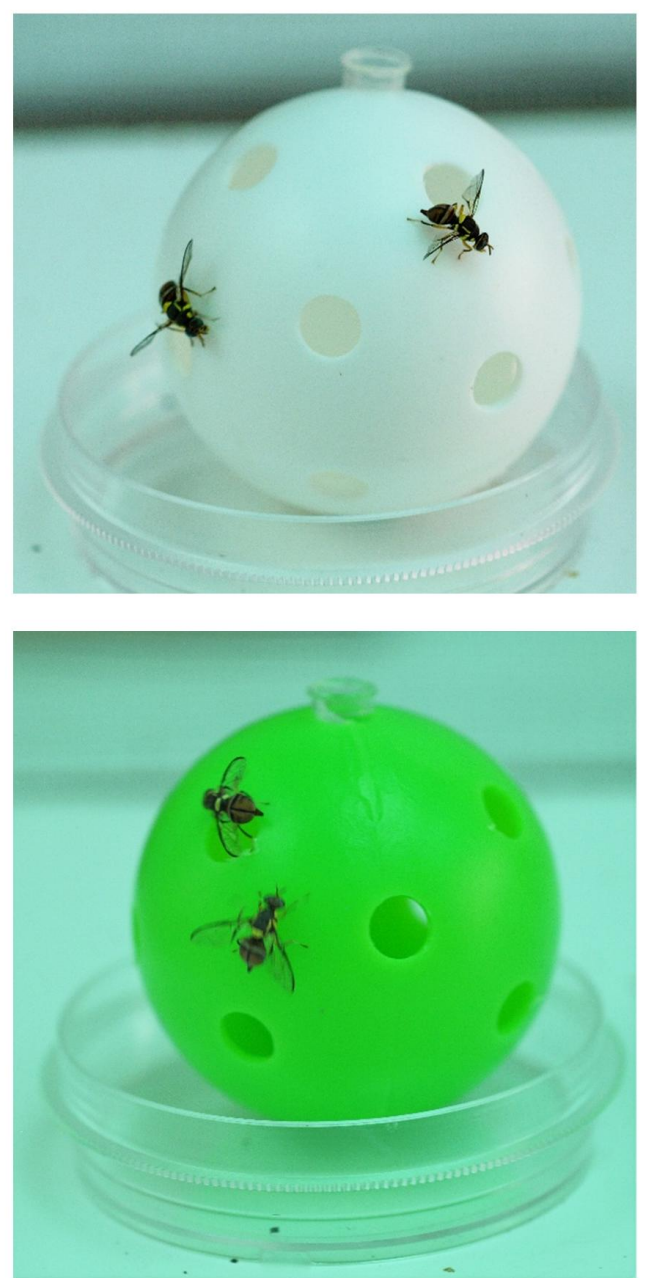
A BdorOR13a MLFNPKPSKDPKNFRFPLQC IWLKLNGSWPLKP - KVTGEFQKYLRLLYSIWAWYVVAMVGITIGF DmOR13a M FYSYP... YKALSFPI Q C VWLKLNGSWPLTESSRPWRSQSLLATAYIVWAWYVIASVGITISY

$$
\text { TM2 }
$$

QSAFLLKSFGN M MVTTENGCTTFMGVLNFVRLLHLRLHQRDFQQLLAQFVKDIWITSSSHPTVER QTAFLLNNLSDIII T TENCCTTFMGVLNFVRLIHLRLNQRKFRQLIENFS Y I I I PNSSKN N VAA TM3

BdorOR13a

DmOR13a

ACARNM RVFQVISVLQSSLITMYCILPLVELYMLTLNVEPDVLDSMPKPFPYKMLFPYDANHG $\overline{W R}$ ECRRRMVTFSIMTSLLACLIIMYCVLPLVEIFFG..... PAFDAQNKPFPYKMIFPYDAQSSWI TM4

BdorOR13a

DmOR13a

- YALTYLFTAWAGVCVVTTLFAEDSLFGFFVSYTCGQFRILHTQIDNIIPDSYAATRAGRGTEVV RYVMTYIFTSYAGICVVTTLFAEDTILGFFITYTCGQFHLLHQRIAGLFAGSNAELAES

BdorOR13a F QREC IRRLDKIANKHCVLFNFVSRMEEFFSP LLLVNFLISSVLICMVGFQLVTGQNMFIGDYVK DmOR13a

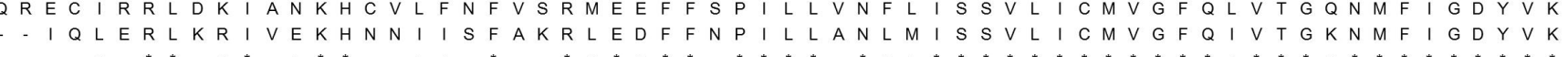
TM6

BdorOR13a

DmOR13a

LVYILSSLSQLFVLCWNGDNTI QNSLEMANHLYACNWESSVKVAADEETKESFPIVSYSTSAAF FI I Y I SSALSQLYVLCENGDALIKQSTLTAQILYECQWEGSDR IEIQQSFT.........

BdorOR13a RKNLQFMIMRSQRQTCITAMK FSILSLNSFSGLISSSMSYFALLQSFYENEEN $R N Q$ I WFM I LCS Q QPVR I TAFKFSTLSLQSFTA I LSTS I S Y F TLLRS VYFDDEKKLD

MPEDLFRIQRNCLRVMGHQDIFDNNEASWSDEQKSKSKRQRWCFRHC QALKYVLLLLFMVSAQLP

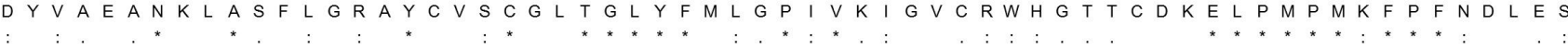

PGYEFAYIYTVFITIVVVMHATSVDGLFVSFTTNLRGHFQALQYFIETNTFDKSEALLQRELGIY PGYEVCFLYTVLVTVVVVAYASAVDGLFISFAINLRAHFQTLQRQIENWEFPSSEPDTQIRLKSI : : * :** :*: :*****:***

TM5

MVVYCTFLSSILLQLL

BdorOR82a

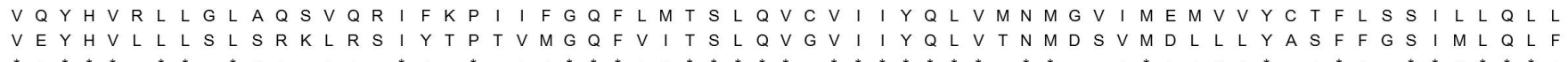

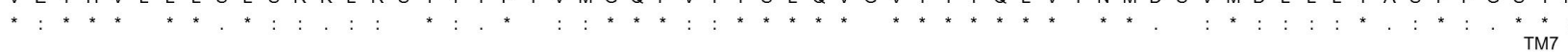

BdorOR82a I YCYGAEFLKTESSAVSTAI QMSQWYNLPPRHRHVLRLMMLRSQREIIISAGFYEASLANFMSIL DmOR82a 
Fig. S4

A

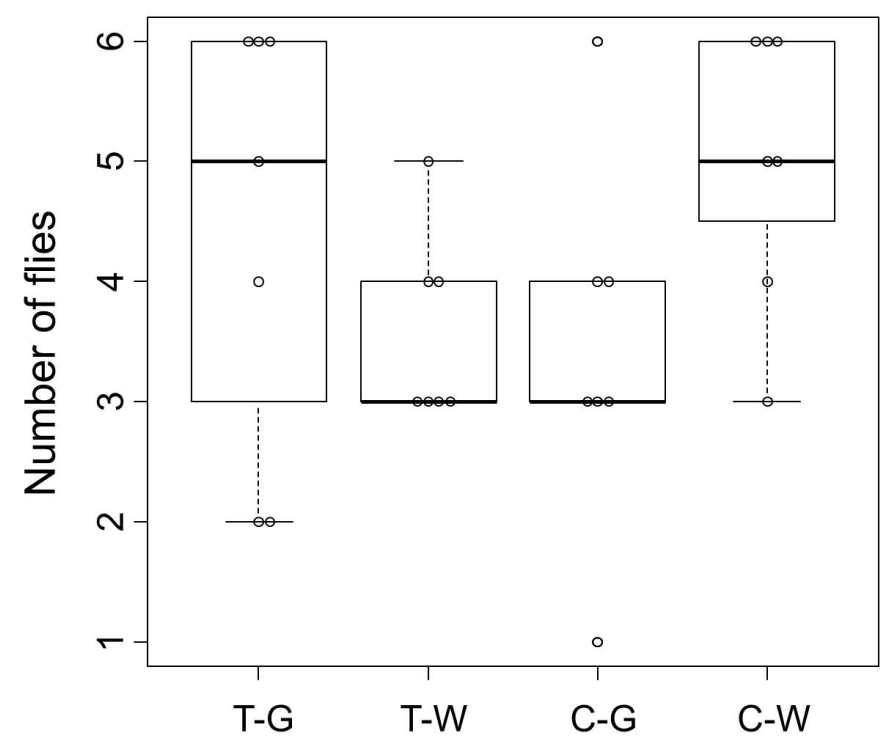

\begin{tabular}{llrrr}
\hline Volatile & Factor & \multicolumn{1}{l}{ AIC } & Deviance & $P$ \\
\hline Ethanol & Null & 105.9 & - & - \\
& Volatile (V) & 107.8 & 0.0853 & 0.770 \\
& Color (C) & 107.7 & 0.237 & 0.626 \\
& VX C & 109.6 & 0.322 & 0.851 \\
\hline
\end{tabular}

B

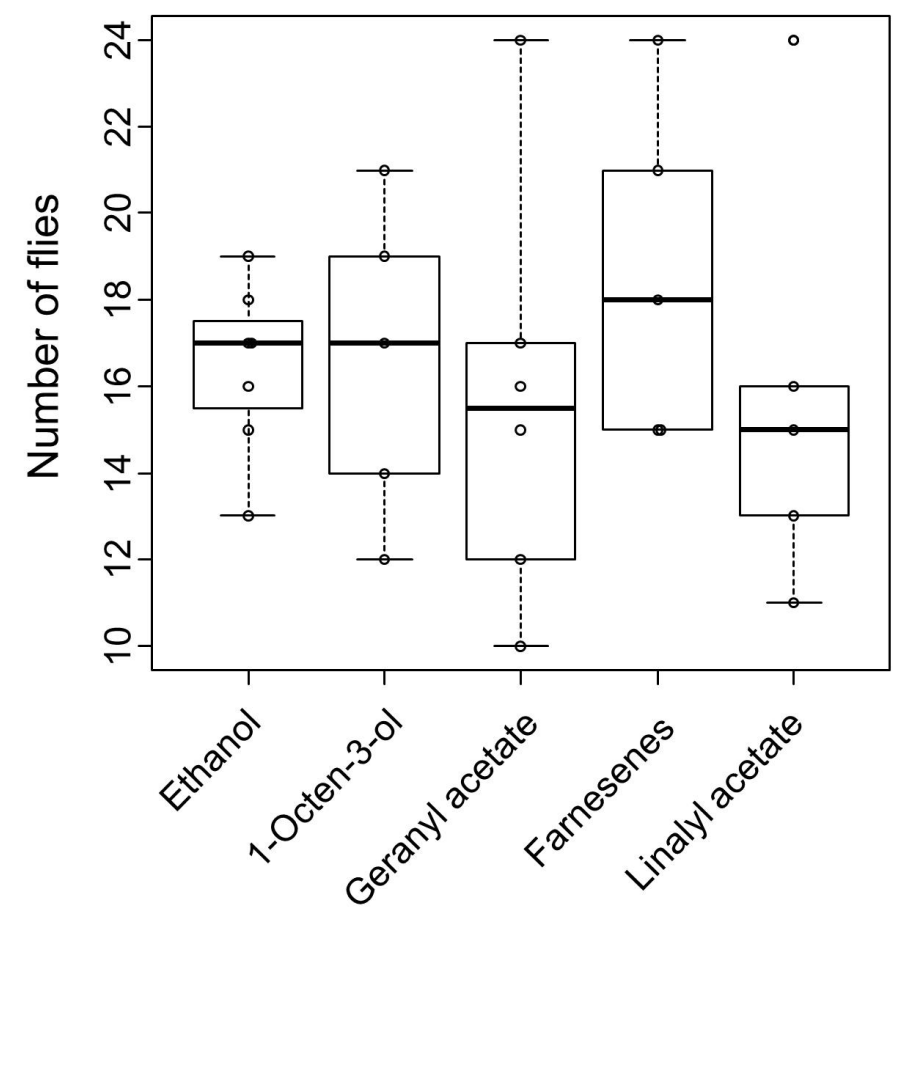

